\title{
On norm closed ideals in $L\left(\ell_{p}, \ell_{q}\right)$
}

\author{
by \\ B. Sari (Denton, TX), Th. Schlumprecht (College Station, TX), \\ N. TOMCZAK-JAEGERMAnn (Edmonton), \\ and V. G. Troitsky (Edmonton)
}

\begin{abstract}
It is well known that the only proper non-trivial norm closed ideal in the algebra $L(X)$ for $X=\ell_{p}(1 \leq p<\infty)$ or $X=c_{0}$ is the ideal of compact operators. The next natural question is to describe all closed ideals of $L\left(\ell_{p} \oplus \ell_{q}\right)$ for $1 \leq p, q<\infty$, $p \neq q$, or equivalently, the closed ideals in $L\left(\ell_{p}, \ell_{q}\right)$ for $p<q$. This paper shows that for $1<p<2<q<\infty$ there are at least four distinct proper closed ideals in $L\left(\ell_{p}, \ell_{q}\right)$, including one that has not been studied before. The proofs use various methods from Banach space theory.
\end{abstract}

1. Introduction. This paper is concerned with the structure of norm closed ideals of the algebra $L(X)$ of all bounded linear operators on an infinite-dimensional Banach space $X$. The classical result of [Calk41] asserts that the only proper non-trivial closed ideal of $L\left(\ell_{2}\right)$ is the ideal of compact operators. The same was shown to be true for $\ell_{p}(1 \leq p<\infty)$ and $c_{0}$ in [GMF60]. It remains open if there are other Banach spaces with only one proper non-trivial closed ideal. The complete structure of closed ideals in $L(X)$ was recently described in [LLR04] for $X=\left(\bigoplus_{n=1}^{\infty} \ell_{2}^{n}\right)_{c_{0}}$ and in [LSZ06] for $X=\left(\bigoplus_{n=1}^{\infty} \ell_{2}^{n}\right)_{\ell_{1}}$. In both cases, there are exactly two nested proper non-zero closed ideals. Apart from those mentioned above, there are no other separable Banach spaces $X$ for which the structure of the closed ideals in $L(X)$ is completely known. The structure of the closed ideals of operators on non-separable Hilbert spaces was independently obtained by Gramsch [Gram67] and Luft [Luft68]. Recently Daws [Daws06] extended

2000 Mathematics Subject Classification: Primary 47L20; Secondary 47B10, 47B37.

Key words and phrases: operator ideal, $\ell_{p}$-space.

The first author was supported by the University of Alberta postdoctoral fellowship. The second author was supported by NSF. The third author holds the Canada Research Chair in Geometric Analysis. The fourth author was supported by the University of Alberta start-up grant. Most of the work on the paper was done during the second author's visit to the University of Alberta in 2003 and during the Workshop on linear analysis and probability at Texas A\&M University in 2004. 
their results to non-separable $\ell_{p}$-spaces, $1 \leq p<\infty$, and non-separable $c_{0}$-spaces.

This motivates the study of the next natural special case $X=\ell_{p} \oplus \ell_{q}$ $(1 \leq p, q<\infty, p \neq q)$, which is our main interest here. There were several results in this direction proved in the 1970's concerning various special ideals or special cases of $p$ and $q$. We refer the reader to the book by Pietsch [Piet78, Chapter 5] for details. In particular, [Piet78, Theorem 5.3.2] asserts that $L\left(\ell_{p} \oplus \ell_{q}\right)$ (with, say, $p<q$ ) has exactly two proper maximal ideals (namely, the ideal of operators which factor through $\ell_{p}$ and the ideals of operators which factor through $\ell_{q}$ ), and establishes a one-to-one correspondence between the non-maximal ideals in the algebra $L\left(\ell_{p} \oplus \ell_{q}\right)$ and the closed "ideals" in $L\left(\ell_{p}, \ell_{q}\right)$. Here an ideal in $L\left(\ell_{p}, \ell_{q}\right)$ means a linear subspace $\mathcal{J}$ of $L\left(\ell_{p}, \ell_{q}\right)$ such that $A T B \in \mathcal{J}$ whenever $A \in L\left(\ell_{q}\right), T \in \mathcal{J}$, and $B \in L\left(\ell_{p}\right)$, and "closed" is always understood with respect to the operator norm topology. Consequently, the subject of the present paper is the structure of closed ideals in $L\left(\ell_{p}, \ell_{q}\right)$ with $1 \leq p \leq q<\infty$.

In this paper we study a number of natural closed ideals in $L\left(\ell_{p}, \ell_{q}\right)$ and relations among them. In particular we show that if $1<p<2<q<\infty$ then the following four closed ideals are proper and distinct: the ideal of all compact operators $\mathcal{K}$, the closed ideal $\mathcal{J}^{I_{p, q}}$ generated by the formal identity operator $I_{p, q}: \ell_{p} \rightarrow \ell_{q}$, the ideal $\mathcal{J}^{\mathrm{FSS}}$ of all finitely strictly singular (FSS) operators, and the closure of the ideal $\mathcal{J}^{\ell_{2}}$ of all $\ell_{2}$-factorable operators (see Section 2 for appropriate definitions). Although these ideals were identified earlier, they were not known to be distinct and proper except for special cases. The following diagram illustrates the relationship between these ideals:

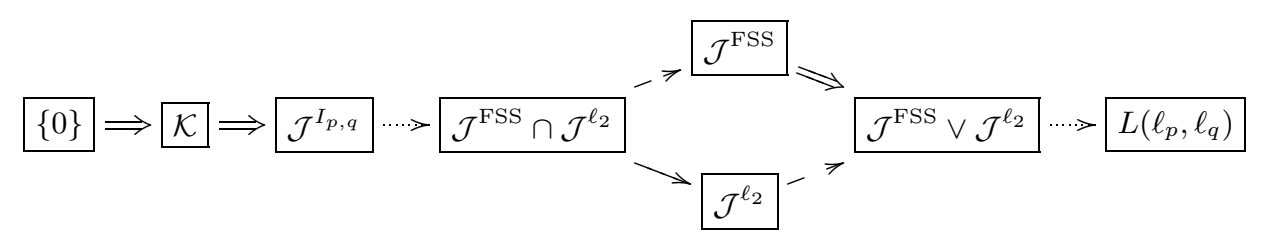

Here arrows stand for inclusions. A solid arrow $(\Rightarrow$ or $\rightarrow$ ) between two ideals means that there are no other ideals sitting properly between the two, while a double arrow coming out of an ideal indicates the only immediate successor. A hyphenated arrow $(-\rightarrow)$ indicates a proper inclusion, while a dotted one indicates that we do not know whether or not the inclusion is proper. In particular, the closed ideals in $L\left(\ell_{p}, \ell_{q}\right)$ are not totally ordered.

The paper is organized as follows. In Section 3 we study the ideal $\mathcal{J}^{I_{p, q}}$ for $1 \leq p<q<\infty$. In [Milm70], Milman proved that $\mathcal{J}^{I_{p, q}}$ is FSS, and therefore, $\mathcal{J}^{I_{p, q}} \subseteq \mathcal{J}^{\mathrm{FSS}}$. Since $\mathcal{J}^{I_{p, q}}$ is not compact, $\mathcal{K}$ is properly contained in $\mathcal{J}^{I_{p, q}}$. We will show that every closed ideal that contains a non-compact operator necessarily contains $\mathcal{J}^{I_{p, q}}$, so that $\mathcal{J}^{I_{p, q}}$ is the least non-compact 
ideal. In Section 4 we consider the ideal $\mathcal{J}^{\ell_{2}}$ when $1<p \leq 2 \leq q<\infty$. We find a specific non-FSS operator $T$ in $\mathcal{J}^{\ell_{2}}$ such that the closed ideal $\mathcal{J}^{T}$ generated by $T$ coincides with $\mathcal{J}^{\ell_{2}}$. This implies, in particular, that $\mathcal{J}^{\text {FSS }}$ is a proper ideal (a result proved in [Milm70] for $p=2 \leq q$ ). Among results on other related ideals we also show that $\mathcal{J}^{\ell_{2}} \subseteq \mathcal{J}^{\ell_{r}}$ for all $r$ between $p$ and $q$, and we prove that every closed ideal of $L\left(\ell_{p}, \ell_{q}\right)$ which contains a non-FSS operator must also contain $\mathcal{J}^{\ell_{2}}$. In Section 5 we consider the "block Hadamard" operator $U$ from $\ell_{p}$ to $\ell_{q}$ for $1<p<2<q<\infty$. We show that $U \notin \mathcal{J}^{\ell_{2}}$, hence $\mathcal{J}^{\ell_{2}}$ is a proper ideal. Since, obviously, $I_{p, q} \in \mathcal{J}^{\ell_{2}}$, it follows that $\mathcal{J}^{I_{p, q}} \subsetneq \mathcal{J}^{U}$. We show in Section 6 that $U$ is FSS, hence $\mathcal{J}^{I_{p, q}} \subsetneq \mathcal{J}^{\text {FSS }}$.

We thank Gilles Pisier for suggesting to us the proof of Theorem 6.5.

2. Notation and preliminaries. We use the standard notation from the Banach space theory as in [LT77, LT79, Tom89, DJT95] and we refer the reader to these books for unexplained notions. Given two Banach spaces $X$ and $Y$, we write $L(X, Y)$ for the space of all continuous linear operators from $X$ to $Y$, and $L(X)$ for $L(X, X)$. A linear subspace $\mathcal{J}$ of $L(X, Y)$ is said to be an ideal if $A T B \in \mathcal{J}$ whenever $A \in L(Y), T \in \mathcal{J}$, and $B \in L(X)$. By a closed ideal we mean an ideal closed in the operator norm topology. We denote by $\mathcal{K}$ the closed ideal of all compact operators.

Throughout this paper, $p$ and $q$ always satisfy $1 \leq p<q<\infty$. We denote by $p^{\prime}$ the conjugate of $p$, that is, $1 / p+1 / p^{\prime}=1$. It is well known (see, e.g., [CPY74]) that $\mathcal{K}$ is contained in every closed ideal of $L\left(\ell_{p}, \ell_{q}\right)$. If $Z$ is a Banach space, we say that an operator $T \in L(X, Y)$ factors through $Z$ if $T=A B$ where $A \in L(Z, Y)$ and $B \in L(X, Z)$; we denote by $\mathcal{J}^{Z}$ the closure of the set of all operators in $L\left(\ell_{p}, \ell_{q}\right)$ that factor through $Z$. It can be easily verified that if $Z$ is isomorphic to $Z \oplus Z$ then $\mathcal{J}^{Z}$ is a subspace, hence an ideal. For $S \in L\left(\ell_{p}, \ell_{q}\right)$ we denote by $\mathcal{J}^{S}$ the closed ideal in $L\left(\ell_{p}, \ell_{q}\right)$ generated by $S$, that is, the smallest closed ideal containing $S$. It is easy to see that $\mathcal{J}^{S}$ consists of operators that can be approximated in norm by operators of the form $\sum_{i=1}^{n} A_{i} S B_{i}$, where $A_{i} \in L\left(\ell_{q}\right)$ and $B_{i} \in L\left(\ell_{p}\right)$ for $i=1, \ldots, n$. If $A$ is an $n \times n$ scalar matrix, we write $\|A\|_{p, q}$ for the norm of $A$ as an operator from $\ell_{p}^{n}$ to $\ell_{q}^{n}$.

It is known that every operator in $L\left(\ell_{p}, \ell_{q}\right)$ is strictly singular (see, e.g., [LT77]). We call an operator $S: X \rightarrow Y$ finitely strictly singular, or $F S S$, if for every $\varepsilon>0$ there exists $n \in \mathbb{N}$ such that $\inf _{x \in E,\|x\|=1}\|S x\|<\varepsilon$ for every $n$-dimensional subspace $E$ of $X$. This class of operators already appeared in [Milm70] where its introduction has been credited to Mityagin and Pełczyński. It can be easily verified (see [Masc94]) that $S$ is FSS if and only if every ultrapower of $S$ is strictly singular. It follows immediately that the set of all FSS operators from $X$ to $Y$ is a closed ideal. Denote by $\mathcal{J}^{\text {FSS }}$ the ideal of all FSS operators in $L\left(\ell_{p}, \ell_{q}\right)$. 
We denote by $\left(e_{i}\right)$ and $\left(f_{i}\right)$ the standard bases of $\ell_{p}$ and $\ell_{q}$ respectively, and we denote their coordinate functionals by $\left(e_{i}^{*}\right)$ and $\left(f_{i}^{*}\right)$. If $\left(x_{n}\right)$ is a sequence in a Banach space, we write $\left[x_{n}\right]$ for its closed linear span. A sequence $\left(x_{n}\right)$ in a Banach space is seminormalized if $\inf _{n}\left\|x_{n}\right\|>0$ and $\sup _{n}\left\|x_{n}\right\|<\infty$.

The following standard lemma is immediately deduced from Propositions 1.a.12 and 2.a.1 of [LT77].

Lemma 2.1. If $X=\ell_{p}(1 \leq p<\infty)$ or $c_{0}$ and $\left(x_{n}\right)$ is a seminormalized sequence in $X$ which converges to zero coordinate-wise (that is, for every $i$, $e_{i}^{*}\left(x_{n}\right) \rightarrow 0$ as $\left.n \rightarrow \infty\right)$, then there is a subsequence $\left(x_{n_{i}}\right)$ equivalent to $\left(e_{i}\right)$ such that $\left[x_{n_{i}}\right]$ is complemented in $X$.

REMARK 2.2. Suppose that $1 \leq p \leq q<\infty$ and $T \in L\left(\ell_{p}, \ell_{q}\right)$. We say that $T$ is block-diagonal if $T=\bigoplus_{n=1}^{\infty} T_{n}$, where $T_{n}: \ell_{p}^{m_{n}} \rightarrow \ell_{q}^{m_{n}}$. Equivalently, there exists a strictly increasing sequence of integers $\left(k_{n}\right)$ such that $T=\sum_{n=1}^{\infty} P_{n} T Q_{n}$, where $Q_{n}$ and $P_{n}$ are the canonical projections from $\ell_{p}$ and $\ell_{q}$ onto the finite-dimensional subspaces spanned by $e_{k_{n}+1}, \ldots, e_{k_{n+1}}$ and $f_{k_{n}+1}, \ldots, f_{k_{n+1}}$ respectively. Note that $m_{n}=k_{n+1}-k_{n}$ and $T_{n}$ can be identified with $P_{n} T Q_{n}$. It can be easily verified that if $p \leq q$ then $\|T\|=\sup _{n}\left\|T_{n}\right\|$. Indeed, $\left\|T_{n}\right\|=\left\|P_{n} T Q_{n}\right\| \leq\|T\|$ as $P_{n}$ and $Q_{n}$ are contractions. On the other hand,

$$
\begin{aligned}
\|T x\| & =\left(\sum_{n=1}^{\infty}\left\|P_{n} T Q_{n} x\right\|^{q}\right)^{1 / q} \leq\left(\sup _{n}\left\|P_{n} T Q_{n}\right\|\right)\left(\sum_{n=1}^{\infty}\left\|Q_{n} x\right\|^{q}\right)^{1 / q} \\
& \leq\left(\sup _{n}\left\|T_{n}\right\|\right)\left(\sum_{n=1}^{\infty}\left\|Q_{n} x\right\|^{p}\right)^{1 / p}=\left(\sup _{n}\left\|T_{n}\right\|\right)\|x\| .
\end{aligned}
$$

Remark 2.3. Suppose that $R \in L\left(\ell_{p}, \ell_{q}\right)$ for $1 \leq p \leq q<\infty$, and $T$ is a block-diagonal submatrix of $R$, that is, $T=\sum_{n=1}^{\infty} P_{n} R Q_{n}$, where $\left(P_{n}\right)$ and $\left(Q_{n}\right)$ are as in Remark 2.2. Then $T$ can be written as a convex combination of operators of the form $U R V$, where $U$ and $V$ are isometries. See Proposition 1.c. 8 of [LT77] and Remark 1 following it for the construction.

3. The formal identity operator $I_{p, q}$. In this section we consider the formal identity operator $I_{p, q}: \ell_{p} \rightarrow \ell_{q}$ for $1 \leq p<q<\infty$. Clearly, $I_{p, q}$ is not compact, so that $\mathcal{K} \subsetneq \mathcal{J}^{I_{p, q}}$. First, we show that $\mathcal{J}^{I_{p, q}}$ is contained in every closed ideal of $L\left(\ell_{p}, \ell_{q}\right)$ except $\mathcal{K}$. This result is probably known to specialists, but we provide a short proof for completeness.

Proposition 3.1. Let $1 \leq p<q<\infty$. If $\mathcal{J}$ is any ideal in $L\left(\ell_{p}, \ell_{q}\right)$ containing a non-compact operator, then $I_{p, q} \in \mathcal{J}$.

Proof. Assume that $\mathcal{J}$ contains a non-compact operator $T$. There exists a normalized sequence $\left(x_{n}\right)$ in $\ell_{p}$ such that $\left(T x_{n}\right)$ has no convergent subse- 
quences. By passing to subsequences and using a standard diagonalization argument, we can assume that $\left(x_{n}\right)$ and $\left(T x_{n}\right)$ converge coordinate-wise. Let $y_{n}=x_{n}-x_{n-1}$; then $\left(y_{n}\right)$ and $\left(T y_{n}\right)$ converge coordinate-wise to zero. Since $\left(T x_{n}\right)$ has no convergent subsequences, we can assume (by passing to a further subsequence if necessary) that $\left(T y_{n}\right)$ is seminormalized. It follows that $\left(y_{n}\right)$ is also seminormalized. Using Lemma 2.1 twice, we can assume (by passing to a subsequence) that $\left(y_{n}\right)$ is equivalent to $\left(e_{i}\right),\left(T y_{n}\right)$ is equivalent to $\left(f_{i}\right)$, and $\left[T y_{n}\right]$ is complemented in $\ell_{q}$.

Let $B: \ell_{p} \rightarrow\left[y_{n}\right]$ be an isomorphism given by $B e_{n}=y_{n}$, and $A:\left[T y_{n}\right] \rightarrow$ $\ell_{q}$ be an isomorphism given by $A\left(T y_{n}\right)=f_{n}$. Since $\left[T y_{n}\right]$ is complemented, $A$ can be extended to an operator on all of $\ell_{q}$. Thus we can view $B$ and $A$ as elements of $L\left(\ell_{p}\right)$ and $L\left(\ell_{q}\right)$ respectively. Observe that $A T B e_{n}=f_{n}$ for each $n$, hence $A T B=I_{p, q}$. It follows that $I_{p, q} \in \mathcal{J}$.

Corollary 3.2. If a closed ideal of $L\left(\ell_{p}, \ell_{q}\right)$ contains a non-compact operator, then it contains $\mathcal{J}^{I_{p, q}}$.

The following result was proved in [Milm70]. For the reader's convenience we provide a short proof.

Proposition 3.3. Suppose that $1 \leq p<q<\infty$. The formal identity operator $I_{p, q}$ is FSS.

We will deduce this proposition from the following lemma, which also appeared in [Milm70].

Lemma 3.4. If $E$ is an n-dimensional subspace of $c_{0}$ then there exists $x \in E$ such that $x$ attains its sup-norm at at least $n$ coordinates.

Proof. The proof is by induction. The statement is trivial for $n=1$. Suppose that it is true for $n$, take any subspace $E$ of $c_{0}$ of dimension $n+1$. By induction hypothesis, there exists $x \in E$ such that

$$
\delta:=\|x\|_{\infty}=\left|x_{i_{1}}\right|=\cdots=\left|x_{i_{n}}\right|
$$

for a set of distinct indices $I=\left\{i_{1}, \ldots, i_{n}\right\}$. Suppose that $\left|x_{i}\right|<\delta$ for all $i \notin I$ (otherwise we are done). Let $Y$ be the subspace of $c_{0}$ consisting of all the sequences that vanish at $i_{1}, \ldots, i_{n}$. Since $Y$ has co-dimension $n$, it follows that $Y \cap E \neq\{0\}$. Pick a non-zero $y \in Y \cap E$. We claim that for some $s>0$ the sequence $x+s y$ attains its sup-norm at at least $n+1$ coordinates. Indeed, $\left|x_{i}+t y_{i}\right|=\delta$ for all $i \in I$ and $t \geq 0$. Consider the function

$$
f(t)=\max _{j \notin I}\left|x_{j}+t y_{j}\right| \text {. }
$$

Clearly, $f$ is continuous, $f(0)<\delta$, and $\lim _{t \rightarrow \infty} f(t)=\infty$. It follows that $f(s)=\delta$ for some $s>0$. Then $\left|x_{i}+s y_{i}\right|=\|x+s y\|_{\infty}=\delta$ for some $i \notin I$.

Proof of Proposition 3.3. Given $\varepsilon>0$, pick $n \in \mathbb{N}$ such that $n^{1 / q-1 / p}<\varepsilon$. Suppose that $E$ is a subspace of $\ell_{p}$ with $\operatorname{dim} E=n$. By Lemma 3.4 there 
exists $x \in E$ and indices $i_{1}, \ldots, i_{n}$ satisfying (1). Without loss of generality, $\|x\|_{p}=1$. It follows that $1=\|x\|_{p}^{p} \geq n \delta^{p}$, so that $\delta \leq n^{-1 / p}$. Then

$$
\|x\|_{q}^{q} \leq\|x\|_{\infty}^{q-p}\|x\|_{p}^{p}=\delta^{q-p} \leq n^{-(q-p) / p}
$$

so that $\|x\|_{q} \leq n^{1 / q-1 / p}<\varepsilon$. It follows that $I_{p, q}$ is FSS.

Corollary 3.5. Let $1 \leq p<q<\infty$. The ideal $\mathcal{K}$ is a proper subset of $\mathcal{J}^{\text {FSS }}$.

4. Operators factorable through $\ell_{2}$. In this section we consider the ideal $\mathcal{J}^{\ell_{2}}$ for $1<p<2<q$. Using Pełczyński's decomposition, we will construct an operator $T: \ell_{p} \rightarrow \ell_{q}$ such that $\mathcal{J}^{\ell_{2}}=\mathcal{J}^{T}$. That is, the closure of the ideal of all $\ell_{2}$-factorable operators is exactly the closed ideal generated by $T$. Furthermore, we show that $T$ fails to be FSS, hence the ideal $\mathcal{J}^{\text {FSS }}$ is proper. It will be obvious from the definition of $T$ that $T$ factors through $\ell_{r}$ whenever $p \leq r \leq q$, so it follows that $\mathcal{J}^{\ell_{2}} \subseteq \mathcal{J}^{\ell_{r}}$. We also show that $T$ factors through every non-FSS operator. It follows that any closed ideal containing a non-FSS operator necessarily contains $\mathcal{J}^{\ell_{2}}$.

To construct $T$, recall that it follows from Pełczyński's decomposition theorem that for every $1<r<\infty, \ell_{r}$ is isomorphic to $\left(\bigoplus_{n=1}^{\infty} \ell_{2}^{n}\right)_{r}$, the $\ell_{r}$-direct sum of $\ell_{2}^{n}$ 's (see [LT77, p. 73]). Let $1<p \leq q<\infty$, put $U: \ell_{p} \rightarrow\left(\bigoplus_{n=1}^{\infty} \ell_{2}^{n}\right)_{p}$ and $V:\left(\bigoplus_{n=1}^{\infty} \ell_{2}^{n}\right)_{q} \rightarrow \ell_{q}$ be two such isomorphisms. By $I_{2, p, q}:\left(\bigoplus_{n=1}^{\infty} \ell_{2}^{n}\right)_{p} \rightarrow$ $\left(\bigoplus_{n=1}^{\infty} \ell_{2}^{n}\right)_{q}$ we denote the formal identity operator, that is, just the change of the norm on the direct sum. Then let $T=V I_{2, p, q} U$, that is,

$$
T: \ell_{p} \stackrel{U}{\longrightarrow}\left(\bigoplus_{n=1}^{\infty} \ell_{2}^{n}\right)_{p} \stackrel{I_{2, p, q}}{\longrightarrow}\left(\bigoplus_{n=1}^{\infty} \ell_{2}^{n}\right)_{q} \stackrel{V}{\longrightarrow} \ell_{q} .
$$

We will call $T$ a Pełczyński decomposition operator.

REMARK 4.1. Note that $T$ is not unique, it is defined up to the isomorphisms $U$ and $V$, so that we have actually constructed a class of operators. It is clear, however, that any two Pełczyński decomposition operators factor through each other. Moreover, one can easily verify that if in the preceding construction we "skip" some of the blocks, that is, if we consider $\left(\bigoplus_{n=1}^{\infty} \ell_{2}^{k_{n}}\right)$ for some strictly increasing sequence of indices $k_{n}$, then the resulting operator $T^{\prime}$ obviously factors through $T$. Conversely, $T$ factors through $T^{\prime}$ because $\ell_{2}^{n}$ is a complemented subspace of $\ell_{2}^{k_{n}}$.

Furthermore, let $E_{n}=U^{-1}\left(\ell_{2}^{n}\right) \subset \ell_{p}$ be the pre-image of the $n$th block of $\left(\bigoplus \ell_{2}^{n}\right)_{p}$. Similarly, put $F_{n}=V\left(\ell_{2}^{n}\right) \subset \ell_{q}$. Then $d\left(E_{n}, \ell_{2}^{n}\right) \leq\|U\| \cdot\left\|U^{-1}\right\|$ and $d\left(F_{n}, \ell_{2}^{n}\right) \leq\|V\| \cdot\left\|V^{-1}\right\|$, where $d(X, Y)$ stands for the Banach-Mazur distance between $X$ and $Y$. Hence, $\left(E_{n}\right)$ and $\left(F_{n}\right)$ are sequences of uniformly Euclidean subspaces of $\ell_{p}$ and $\ell_{q}$ respectively. Note that $T\left(E_{n}\right)=F_{n}$, so that 
$T$ fixes copies of $\ell_{2}^{n}$ for all $n \in \mathbb{N}$. This immediately implies the following result.

Proposition 4.2. For $1<p \leq q<\infty$, every Pełczyński decomposition operator fails to be FSS.

Corollary 4.3. For $1<p \leq q<\infty$, the ideal $\mathcal{J}^{\mathrm{FSS}}$ is proper.

Our next goal is to show that if $1<p \leq 2 \leq q<\infty$ then $\mathcal{J}^{T}=\mathcal{J}^{\ell_{2}}$. We will make use of the concept of $\ell_{2}$-factorable norm $\gamma_{2}$. Recall that if $S \in L(X, Y)$ ( $X$ and $Y$ Banach spaces) then $\gamma_{2}(S)=\inf \left\|S_{1}\right\|\left\|S_{2}\right\|$, where the infimum is taken over all factorizations $S=S_{1} S_{2}$ where $S_{2}: X \rightarrow \ell_{2}$ and $S_{1}: \ell_{2} \rightarrow Y$. It is known that $\gamma_{2}$ is a norm on the ideal of all $\ell_{2}$-factorable operators, and $\gamma_{2}(A S B) \leq\|A\| \gamma_{2}(S)\|B\|$ whenever $X \stackrel{B}{\rightarrow} X \stackrel{S}{\rightarrow} Y \stackrel{A}{\rightarrow} Y$. See [Tom89, DJT95] for more information on $\gamma_{2}$.

Lemma 4.4. Suppose that $R \in L\left(\ell_{p}, \ell_{q}\right), 1<p \leq q<\infty$, and $\varepsilon>0$.

(i) There exist two block-diagonal operators $V, W \in L\left(\ell_{p}, \ell_{q}\right)$ such that $\|W\| \leq\|R\|+\varepsilon,\|V\| \leq 2\|R\|+2 \varepsilon$, and $\|R-(W+V)\|<\varepsilon$.

(ii) Suppose that, in addition, $R$ is $\ell_{2}$-factorable. Then $V$ and $W$ can be chosen to be $\ell_{2}$-factorable, and $\gamma_{2}(W) \leq \gamma_{2}(R)+\varepsilon, \gamma_{2}(V) \leq$ $2 \gamma_{2}(R)+2 \varepsilon$, and $\gamma_{2}(R-(W+V))<\varepsilon$.

Proof. Let $r_{i, j}$ stand for the $(i, j)$ th entry of the matrix of $R$, that is, $r_{i, j}=f_{i}^{*}\left(R e_{j}\right)$. For the purpose of this proof we introduce the following notation: for $\Omega \subset \mathbb{N} \times \mathbb{N}$, we define the matrix $R_{\Omega}=\left(\varrho_{i, j}\right)$ by

$$
\varrho_{i, j}= \begin{cases}r_{i, j} & \text { if }(i, j) \in \Omega \\ 0 & \text { otherwise. }\end{cases}
$$

We start by approximating $R$ by a matrix $S$ with finitely many entries in every row and every column. Namely, by truncating each row and each column of $R$ sufficiently far we can find two strictly increasing sequences $\left(M_{j}\right)$ and $\left(N_{i}\right)$ of positive integers such that $\left\|R-R_{\Gamma}\right\|<\varepsilon$ where $\Gamma \subseteq \mathbb{N} \times \mathbb{N}$ is defined by

$$
(i, j) \in \Gamma \quad \text { iff } \quad i \leq M_{j} \text { and } j \leq N_{i} .
$$

Put $S=R_{\Gamma}$.

We will define two strictly increasing sequences $\left(k_{n}\right)$ and $\left(l_{n}\right)$ of positive integers such that $\Gamma$ is contained in the union of two block-diagonal sets $\Delta=\bigcup_{n=1}^{\infty} \Delta_{n}$ and $\Lambda=\bigcup_{n=1}^{\infty} \Lambda_{n}$ where

$$
\begin{aligned}
\Delta_{n} & =\left\{(i, j) \in \Gamma \mid k_{n-1}<i, j \leq k_{n}\right\}, \\
\Lambda_{n} & =\left\{(i, j) \in \Gamma \mid l_{n-1}<i, j \leq l_{n}\right\} .
\end{aligned}
$$

We define the sequences $\left(k_{n}\right)$ and $\left(l_{n}\right)$ by an interlaced induction. Put $k_{0}=0$, 
$l_{0}=1$. For $n \geq 0$ we let

$$
k_{n+1}=\max \left\{M_{l_{n}}, N_{l_{n}}\right\}, \quad l_{n+1}=\max \left\{M_{k_{n+1}}, N_{k_{n+1}}\right\} .
$$

Clearly, $\left(k_{n}\right)$ and $\left(l_{n}\right)$ are strictly increasing. Next, we show that $\Gamma \subseteq \Delta \cup \Lambda$. Let $(i, j) \in \Gamma$. There exists $n$ such that $l_{n}<\max \{i, j\} \leq l_{n+1}$. If $l_{n}<$ $\min \{i, j\}$, then $l_{n}<i, j \leq l_{n+1}$, so that $(i, j) \in \Lambda$. Suppose now that $\min \{i, j\} \leq l_{n}$. Then either $i$ or $j$ is less than or equal to $l_{n}$, while the other is greater than $l_{n}$. Say, $i \leq l_{n}$ and $j>l_{n}$. It follows that

$$
i \leq l_{n} \leq N_{l_{n}} \leq k_{n+1} \quad \text { and } \quad j>l_{n} \geq N_{k_{n}} \geq k_{n} .
$$

Therefore $j \leq N_{i} \leq N_{l_{n}} \leq k_{n+1}$. Also, $N_{i} \geq j>l_{n} \geq N_{k_{n}}$ yields $i>k_{n}$. Hence, $k_{n}<i, j \leq k_{n+1}$, so that $(i, j) \in \Delta$.

Set $W=S_{\Delta}$ and $V=S-W$. Then the non-zero entries of $W$ and $V$ are located in $\Delta$ and $\Lambda$ respectively, so that $W$ and $V$ are block-diagonal. By the definition of $S$ we have $\|R-(W+V)\|<\varepsilon$. Since $W$ is a block-diagonal part of $S$, Remark 2.3 yields $\|W\| \leq\|S\| \leq\|R\|+\varepsilon$. Finally, it follows from $V=S-W$ that $\|V\| \leq 2\|R\|+2 \varepsilon$.

If $R$ is $\ell_{2}$-factorable, then we can choose $S$ with finitely many entries in each row and column such that $S$ is also $\ell_{2}$-factorable and $\gamma_{2}(R-S)<\varepsilon$. Indeed, let $R=R_{1} R_{2}$ be a factorization of $R$ through $\ell_{2}$. Approximate $R_{1}$ and $R_{2}$ in norm by $S_{1}$ and $S_{2}$, respectively, such that $S_{1}$ and $S_{2}$ have finitely many entries in every row and column. Put $S=S_{1} S_{2}$; then $S$ is as claimed. We use the triangle inequality to show that $\gamma_{2}(R-S)<\varepsilon$ when $\left\|R_{1}-S_{1}\right\|$ and $\left\|R_{2}-S_{2}\right\|$ are sufficiently small.

Set $W=S_{\Delta}$ and $V=S-W$. It follows from Remark 2.3 that $\gamma_{2}(W) \leq$ $\gamma_{2}(S) \leq \gamma_{2}(R)+\varepsilon$. Then $\gamma_{2}(V)=\gamma_{2}(S-W) \leq 2 \gamma_{2}(R)+2 \varepsilon$. In particular, $W$ and $V$ are $\ell_{2}$-factorable.

REMARK 4.5. In a similar fashion one can show that every operator between two Banach spaces with shrinking unconditional bases can be approximated by a sum of two block-diagonal operators.

REMARK 4.6. A slight modification of the proof Lemma 4.4(i) yields $\|W\| \leq\|R\|$. Indeed, choose $M_{j}$ and $N_{i}$ sufficiently large so that not only $\left\|R-R_{\Gamma}\right\|<\varepsilon$ but also $\left\|R-R_{\Omega}\right\|<\varepsilon$ for every $\Omega \subseteq \mathbb{N} \times \mathbb{N}$ such that $\Gamma \subseteq \Omega$. Then, after constructing $\Delta$ and $\Lambda$, put $W=R_{\Delta}$ and $V=R_{\Delta \cup \Lambda}-W$. Then the non-zero entries of $W$ and $V$ are located in $\Delta$ and $\Lambda$, respectively, so that $W$ and $V$ are block-diagonal. Since $\Delta \cup \Lambda \supseteq \Gamma$ we have $\|R-(W+V)\|<\varepsilon$. Since $W$ is a block-diagonal part of $R$, Remark 2.3 yields $\|W\| \leq\|R\|$. Finally, $\|V\| \leq\left\|R_{\Delta \cup \Lambda}\right\|+\|W\| \leq 2\|R\|+\varepsilon$.

Theorem 4.7. If $1<p \leq 2 \leq q$ and $T$ is a Petczyński decomposition operator, then $\mathcal{J}^{T}=\mathcal{J}^{\ell_{2}}$. 
Proof. Observe that $I_{2, p, q}$, being the formal identity from $\left(\bigoplus_{n=1}^{\infty} \ell_{2}^{n}\right)_{p}$ to $\left(\bigoplus_{n=1}^{\infty} \ell_{2}^{n}\right)_{q}$, factors through $\left(\bigoplus_{n=1}^{\infty} \ell_{2}^{n}\right)_{2}=\ell_{2}$. It follows that $T$ factors through $\ell_{2}$ and, therefore, $\mathcal{J}^{T} \subseteq \mathcal{J}^{\ell_{2}}$.

We show that $\mathcal{J}^{\ell_{2}} \subseteq \mathcal{J}^{T}$. Clearly, it suffices to show that every $\ell_{2^{-}}$ factorable operator belongs to $\mathcal{J}^{T}$. In view of Lemma 4.4(ii), it suffices to show this for block-diagonal operators. Let $W$ be an $\ell_{2}$-factorable blockdiagonal operator. Then we can write $W=\bigoplus_{n=1}^{\infty} A_{n} B_{n}$, where $B_{n}$ : $\ell_{p}^{k_{n}} \rightarrow \ell_{2}^{k_{n}}$ and $A_{n}: \ell_{2}^{k_{n}} \rightarrow \ell_{q}^{k_{n}}$ such that $\sup _{n}\left\|A_{n}\right\|$ and $\sup _{n}\left\|B_{n}\right\|$ are finite. By merging consecutive blocks if necessary, we can assume without loss of generality that $\left(k_{n}\right)$ is strictly increasing. Observe that the operators

$$
B=\bigoplus_{n=1}^{\infty} B_{n}:\left(\bigoplus_{n=1}^{\infty} \ell_{p}^{k_{n}}\right)_{p} \rightarrow\left(\bigoplus_{n=1}^{\infty} \ell_{2}^{k_{n}}\right)_{p}
$$

and

$$
A=\bigoplus_{n=1}^{\infty} A_{n}:\left(\bigoplus_{n=1}^{\infty} \ell_{2}^{k_{n}}\right)_{q} \rightarrow\left(\bigoplus_{n=1}^{\infty} \ell_{q}^{k_{n}}\right)_{q}
$$

are bounded, and $W=A I_{0} B$, with $I_{0}$ the formal identity from $\left(\bigoplus_{n=1}^{\infty} \ell_{2}^{k_{n}}\right)_{p}$ to $\left(\bigoplus_{n=1}^{\infty} \ell_{q}^{k_{n}}\right)_{q}$. Thus, $W$ factors through $I_{0}$. It follows from Remark 4.1 that $I_{0}$ factors through $T$. Hence, $W$ factors through $T$.

REMARK 4.8. Actually, we proved that every operator in $\mathcal{J}^{\ell_{2}}$ can be approximated by sums of two $T$-factorable operators.

REMARK 4.9. Suppose that $1<p<r<q$. Then $I_{2, p, q}$ in (2) factors through $\left(\bigoplus_{n=1}^{\infty} \ell_{2}^{n}\right)_{r}$, which is isomorphic to $\ell_{r}$. It follows that $T$ factors through $\ell_{r}$. Then Theorem 4.7 implies that $\mathcal{J}^{\ell_{2}} \subseteq \mathcal{J}^{\ell_{r}}$ when $p \leq 2 \leq q$.

Next, we show that if $p<2<q$ then $\mathcal{J}^{\ell_{2}}$ is the least closed ideal beyond $\mathcal{J}^{\text {FSS }}$, that is, every closed ideal that contains a non-FSS operator also contains $\mathcal{J}^{\ell_{2}}$. For the proof we need the following well-known fact.

THEOREM 4.10. For every $1<r<\infty$ there exists $K>0$ such that for all $n \in \mathbb{N}$ there exists $N \in \mathbb{N}$ such that every $N$-dimensional subspace $F \subset \ell_{r}$ contains an $n$-dimensional subspace $E$ which is $K$-complemented in $\ell_{r}$ and 2-isomorphic to $\ell_{2}^{n}$.

REMARK 4.11. The theorem follows by simultaneous use of Dvoretzky's theorem both in a subspace $F \subset \ell_{r}$ and in its dual $F^{*}$ (see, e.g., [MS86]). This gives the result with $N=C n^{r / 2}$ and $K=C^{\prime} \sqrt{\max \left\{r, r^{\prime}\right\}}$, where $C, C^{\prime}>0$ are absolute constants. This theorem can also be viewed, for example, as a special case of results in [FT79].

We will also routinely use the following observation. 
REMARK 4.12. Suppose that $\left(E_{n}\right)$ is a sequence of subspaces of a Banach space $X$ which are uniformly Euclidean and uniformly complemented in $X$. That is, there exist a constant $C>0$ and sequences $\left(P_{n}\right)$ and $\left(V_{n}\right)$ such that $P_{n}$ is a projection from $X$ onto $E_{n}$ with $\left\|P_{n}\right\|<C$, and $V_{n}: E_{n} \rightarrow \ell_{2}^{n}$ is an isomorphism with $\left\|V_{n}\right\| \cdot\left\|V_{n}^{-1}\right\| \leq C$ for every $n$. Let $G_{n}$ be a subspace of $E_{n}(n \in \mathbb{N})$. Then it is easy to see that the $G_{n}$ 's are uniformly Euclidean and uniformly complemented in $X$ as well.

For $x \in \ell_{r}$ we write $\operatorname{supp} x=\left\{i \in \mathbb{N} \mid x_{i} \neq 0\right\}$. For $A \subseteq \ell_{r}$ put $\operatorname{supp} A=\bigcup_{x \in A} \operatorname{supp} x$.

TheOREM 4.13. Let $1<p \leq 2 \leq q<\infty$. If $R \in L\left(\ell_{p}, \ell_{q}\right)$ is not FSS, then every Pełczyński decomposition operator factors through $R$.

Proof. Since $R$ is not FSS, there exist a constant $C>0$ and a sequence $\left(E_{n}\right)$ of subspaces of $\ell_{p}$ such that $\operatorname{dim} E_{n} \rightarrow \infty$ as $n \rightarrow \infty$, and $R_{\mid E_{n}}$ is invertible with $\left\|\left(R_{\mid E_{n}}\right)^{-1}\right\| \leq C$. We can assume, in addition, that $\operatorname{supp} E_{n}$ is finite by truncating all the vectors in a basis of $E_{n}$ sufficiently far (and adjusting $C$ if necessary). Let $F_{n}=R\left(E_{n}\right)$. Using Theorem 4.10 and Remark 4.12 we can easily obtain subspaces $E_{n}^{\prime} \subset E_{n}$ and $F_{n}^{\prime} \subset F_{n}$ which are $C$-Euclidean, $C$-complemented in $\ell_{p}$ and $\ell_{q}$ respectively, and such that $F_{n}^{\prime}=R\left(E_{n}^{\prime}\right)$. By passing to a subsequence we may assume that $\operatorname{dim} E_{n}^{\prime}=n$, and we relabel the sequences so obtained as $\left(E_{n}\right)$ and $\left(F_{n}\right)$. Let $Q_{n}: \ell_{q} \rightarrow F_{n}$ be a projection with $\left\|Q_{n}\right\| \leq C$.

We are going to define sequences $\left(\widehat{E}_{n}\right),\left(\widehat{F}_{n}\right)$ and $\left(\widehat{Q}_{n}\right)$ which satisfy all the properties described in the previous paragraph and, in addition, there exists a strictly increasing sequence $\left(m_{n}\right)$ in $\mathbb{N}$ such that the following four conditions are satisfied:

(i) $m_{n-1}<\min \operatorname{supp} \widehat{E}_{n}$ and $m_{n-1}<\min \operatorname{supp} \widehat{F}_{n}$;

(ii) $\widehat{Q}_{n} y=0$ whenever maxsupp $y \leq m_{n-1}$;

(iii) $m_{n} \geq \max \operatorname{supp} \widehat{E}_{n}$;

(iv) $\left\|\widehat{Q}_{n} y\right\| \leq 2^{-n}\|y\|$ whenever min supp $y>m_{n}$.

We construct the sequences inductively. Let $m_{0}=0$, and suppose that we already constructed $\widehat{E}_{i}, \widehat{F}_{i}, \widehat{Q}_{i}$, and $m_{i}$ for all $i<n$. Let $G$ and $G^{\prime}$ be the subspaces of $\ell_{p}$ and $\ell_{q}$, respectively, consisting of all vectors whose first $m_{n-1}$ coordinates are zero. Put $k=2 m_{n-1}+n$. It follows from $\operatorname{dim} F_{k}=k$ and $\operatorname{codim} G^{\prime}=m_{n-1}$ that $m_{n-1}+n \leq \operatorname{dim} F_{k} \cap G^{\prime}=\operatorname{dim} R^{-1}\left(F_{k} \cap G^{\prime}\right)$, because $R_{\mid E_{k}}$ is an isomorphism. Since $\operatorname{codim} G=m_{n-1}$ we have $G \cap R^{-1}\left(F_{k} \cap G^{\prime}\right)$ $\geq n$. Let $\widehat{E}_{n}$ be an $n$-dimensional subspace of $G \cap R^{-1}\left(F_{k} \cap G^{\prime}\right)$, and $\widehat{F}_{n}=$ $\bar{R}\left(\widehat{E}_{n}\right)$. Then $\widehat{E}_{n} \subseteq G$ and $\widehat{F}_{n} \subseteq G^{\prime}$, hence (i) is satisfied. Clearly, $\widehat{F}_{n}$ is $\widehat{C}$ complemented in $\ell_{q}$, where $\widehat{C}=C^{2}$. Then there exists a projection $Q^{\prime}: \ell_{q} \rightarrow$ $\widehat{F}_{n}$ such that $\left\|Q^{\prime}\right\| \leq \widehat{C}$. Let $\widehat{Q}_{n}=Q^{\prime} P$, where $P$ is the basis projection of $\ell_{q}$ 
onto $\left[f_{i}\right]_{i \geq m_{n-1}}$. Then $\widehat{Q}_{n}$ is again a projection from $\ell_{q}$ onto $\widehat{F}_{n},\left\|\widehat{Q}_{n}\right\| \leq \widehat{C}$, and so (ii) is satisfied. Since $\operatorname{rank} \widehat{Q}_{n}=n$, we can write $\widehat{Q}_{n}=\sum_{j=1}^{n} z_{j} \otimes z_{j}^{*}$, where $z_{1}, \ldots, z_{j} \in \ell_{p}$ and $z_{1}^{*}, \ldots, z_{j}^{*} \in \ell_{q}^{*}$. Then we can find $r \in \mathbb{N}$ sufficiently large such that if $\|y\| \leq 1$ and minsupp $y>r$ then $\left|z_{j}^{*}(y)\right|$ is sufficiently small for all $j=1, \ldots, n$, so that $\|\widehat{Q} y\| \leq 2^{-n}$. Let $m_{n}=\max \{r, s\}$, where $s=\max \operatorname{supp} \widehat{E}_{n}$; then (iii) and (iv) are satisfied.

For convenience, we relabel $\widehat{E}_{n}, \widehat{F}_{n}, \widehat{Q}_{n}$, and $\widehat{C}$ as $E_{n}, F_{n}, Q_{n}$, and $C$ again. For every $n$ suppose that $V_{n}$ is a $C$-isomorphism of $\ell_{2}^{n}$ onto $E_{n}$ with $\left\|V_{n}\right\|=1$ and $\left\|V_{n}^{-1}\right\| \leq C$. Put

$$
V=\bigoplus_{n=1}^{\infty} V_{n}:\left(\bigoplus_{n=1}^{\infty} \ell_{2}^{n}\right)_{p} \rightarrow\left(\bigoplus_{n=1}^{\infty} E_{n}\right)_{p}
$$

Since the $E_{n}$ 's are disjointly supported, we can consider $\left(\bigoplus_{n=1}^{\infty} E_{n}\right)_{p}$ as a subspace of $\ell_{p}$. It follows that $V$ is a $C$-isomorphism between $\left(\bigoplus_{n=1}^{\infty} \ell_{2}^{n}\right)_{p}$ and a subspace of $\ell_{p}$. Define

$$
W: \ell_{q} \rightarrow\left(\bigoplus_{n=1}^{\infty} \ell_{2}^{n}\right)_{q} \quad \text { via } \quad x \mapsto\left(V_{n}^{-1}\left(R_{\mid E_{n}}\right)^{-1} Q_{n} x\right)_{n=1}^{\infty} .
$$

We claim that $W$ is bounded. Indeed, pick $x \in \ell_{q}$. Then

$$
\|W x\|=\left(\sum_{n=1}^{\infty}\left\|V_{n}^{-1}\left(R_{\mid E_{n}}\right)^{-1} Q_{n} x\right\|_{2}^{q}\right)^{1 / q} \leq C^{2}\left(\sum_{n=1}^{\infty}\left\|Q_{n} x\right\|^{q}\right)^{1 / q} .
$$

Let $P_{k}$ be the basis projection from $\ell_{q}$ onto $\left[f_{i}\right]_{i=m_{k-1}+1}^{m_{k}}$. Then $x=\sum_{k=1}^{\infty} P_{k} x$. It follows from (ii) that $Q_{n} P_{k} x=0$ whenever $k<n$. Furthermore, (iv) yields $\left\|Q_{n}\left(\sum_{k>n} P_{k} x\right)\right\| \leq 2^{-n}\|x\|$. Also, $\left\|Q_{n} P_{n} x\right\| \leq C\left\|P_{n} x\right\|$. Therefore, $\left\|Q_{n} x\right\| \leq C\left\|P_{n} x\right\|+2^{-n}\|x\|$. Using the Cauchy-Schwarz inequality, we get

$$
\left(\sum_{n=1}^{\infty}\left\|Q_{n} x\right\|^{q}\right)^{1 / q} \leq\left(\sum_{n=1}^{\infty}\left(C\left\|P_{n} x\right\|\right)^{q}\right)^{1 / q}+\left(\sum_{n=1}^{\infty}\left(2^{-n}\|x\|\right)^{q}\right)^{1 / q} \leq(C+1)\|x\| .
$$

Together with (3) this shows that $W$ is bounded.

Finally, it is easy to see that $W R V=I_{2, p, q}$; it follows easily that every Pełczyński decomposition operator factors through $R$.

Corollary 4.14. Let $1<p \leq 2 \leq q<\infty$. If $R \in L\left(\ell_{p}, \ell_{q}\right)$ is not FSS, then $\mathcal{J}^{\ell_{2}} \subseteq \mathcal{J}^{R}$.

5. Operators not factorable through $\ell_{2}$. We employ the following known theorem (see [DJT95, Theorem 9.13] or [Tom89, Theorem 27.1]) to deduce conditions for an operator in $L\left(\ell_{p}, \ell_{q}\right)$ to factor through $\ell_{r}$.

Theorem 5.1. Let $1 \leq r<\infty$, let $U: X \rightarrow Y$ be a bounded linear operator between Banach spaces $X$ and $Y$, and let $C \geq 0$. The following are equivalent: 
(i) There exists a subspace $L$ of $L_{r}(\mu), \mu$ a measure, and a factorization $U=V W$, where $V: L \rightarrow Y$ and $W: X \rightarrow L$ are bounded linear operators with $\|V\| \cdot\|W\| \leq C$.

(ii) Whenever finite sequences $\left(x_{i}\right)_{i=1}^{n}$ and $\left(z_{i}\right)_{i=1}^{m}$ in $X$ satisfy

$$
\sum_{i=1}^{m}\left|\left\langle x^{*}, z_{i}\right\rangle\right|^{r} \leq \sum_{i=1}^{n}\left|\left\langle x^{*}, x_{i}\right\rangle\right|^{r} \quad \text { for all } x^{*} \in X^{*},
$$

then

$$
\sum_{i=1}^{m}\left\|U z_{i}\right\|^{r} \leq C^{r} \sum_{i=1}^{n}\left\|x_{i}\right\|^{r} .
$$

Let us use Theorem 5.1 to state a criterion for an operator $U: \ell_{p}^{m} \rightarrow \ell_{q}^{m}$ not to factor as $U=A B$ with $\|B\|_{p, r} \cdot\|A\|_{r, q} \leq C$.

Corollary 5.2. Let $m \in \mathbb{N}, C>1$, and $r>1$, and assume that $U$ is an invertible $m \times m$ matrix. Let $\delta=\left\|U^{-1}\right\|_{\widetilde{r}^{\prime}, r^{\prime}}$. Then $\|B\|_{p, r}\|A\|_{r, q} \geq \delta^{-1}$ for any factorization $U=A B$. Moreover, if $\widetilde{U}$ is another $m \times m$ matrix with

$$
\|\widetilde{U}-U\|_{p, q} \leq\left(2 \max _{1 \leq i \leq m}\left\|U^{-1} e_{i}\right\|_{p}\right)^{-1},
$$

then $\|B\|_{p, r}\|A\|_{r, q} \geq(2 \delta)^{-1}$ for any factorization $\widetilde{U}=A B$.

Proof. For $i=1, \ldots, m$ let $x_{i}=e_{i}$ and $z_{i}=\delta^{-1} U^{-1} e_{i}$ and observe that for any $x^{*} \in \mathbb{R}^{m}$,

$$
\begin{aligned}
\left(\sum_{i=1}^{m}\left|\left\langle x^{*}, z_{i}\right\rangle\right|^{r}\right)^{1 / r} & =\delta^{-1}\left(\sum_{i=1}^{m}\left|\left\langle\left(U^{-1}\right)^{*} x^{*}, e_{i}\right\rangle\right|^{r}\right)^{1 / r}=\delta^{-1}\left\|\left(U^{-1}\right)^{*} x^{*}\right\|_{r} \\
& \leq \delta^{-1}\left\|U^{-1}\right\|_{r^{\prime}, r^{\prime}}\left\|x^{*}\right\|_{r}=\left(\sum_{i=1}^{m}\left|\left\langle x^{*}, x_{i}\right\rangle\right|^{r}\right)^{1 / r}
\end{aligned}
$$

which implies that the hypothesis of (ii) in Theorem 5.1 is satisfied. Secondly it follows that

$$
\sum_{i=1}^{m}\left\|U z_{i}\right\|_{q}^{r}=\delta^{-r} m=\delta^{-r} \sum_{i=1}^{m}\left\|x_{i}\right\|_{p}^{r},
$$

which means that the conclusion of (ii) in Theorem 5.1 is not satisfied for any $C<\delta^{-1}$. It follows that condition (i) in Theorem 5.1 fails whenever $C<\delta^{-1}$.

Now assume that $\widetilde{U}$ is another $m \times m$ matrix satisfying (4), then it follows for $i=1, \ldots, m$ that

$$
\begin{aligned}
\left\|\widetilde{U}\left(z_{i}\right)\right\|_{q} & \geq\left\|U\left(z_{i}\right)\right\|_{q}-\left\|(U-\widetilde{U})\left(z_{i}\right)\right\|_{q} \\
& \geq \frac{1}{2}\left\|U\left(z_{i}\right)\right\|_{q}+\left(\frac{1}{2}\left\|U\left(z_{i}\right)\right\|_{q}-\|U-\widetilde{U}\|_{p, q}\left\|z_{i}\right\|_{p}\right) \\
& =\frac{1}{2}\left\|U\left(z_{i}\right)\right\|_{q}+\left(\frac{1}{2 \delta}-\|U-\widetilde{U}\|_{p, q} \delta^{-1}\left\|U^{-1} e_{i}\right\|_{p}\right) \geq \frac{1}{2}\left\|U\left(z_{i}\right)\right\|_{q},
\end{aligned}
$$


which implies, together with (5), that for $\widetilde{U}$ the conclusion of (ii) in Theorem 5.1 is not satisfied for any $C<\delta^{-1} / 2$, hence (i) fails in this case.

We will now define an operator which will be crucial for the rest of the paper, and we start with the following notations. Let $H_{n}$ be the $n$th Hadamard matrix. That is,

$$
H_{1}=(1), \quad H_{n+1}=\left(\begin{array}{cc}
H_{n} & H_{n} \\
H_{n} & -H_{n}
\end{array}\right) \quad \text { for every } n \geq 1 .
$$

Then $H_{n}$ is an $N \times N$ matrix where $N=2^{n}$. We use the identifications $\ell_{p}=\left(\bigoplus_{n=1}^{\infty} X_{n}\right)_{p}$ and $\ell_{q}=\left(\bigoplus_{n=1}^{\infty} Y_{n}\right)_{q}$, where $X_{n}=\ell_{p}^{2^{n}}$ and $Y_{n}=\ell_{q}^{2^{n}}$ are block subspaces of $\ell_{p}$ and $\ell_{q}$ respectively. We consider $H_{n}$ as an operator from $X_{n}$ to $Y_{n}$. Put

$$
U_{n}=N^{-1 / \min \left\{p^{\prime}, q\right\}} H_{n} \quad \text { where } N=2^{n}, \quad \text { and } \quad U=\bigoplus_{n=1}^{\infty} U_{n}: \ell_{p} \rightarrow \ell_{q} .
$$

REMARK 5.3. Observe that $N^{-1 / 2} H_{n}$ is a unitary matrix on $\ell_{2}^{N}$. In particular, it is an isometry on $\ell_{2}^{N}$, hence $\left\|H_{n}\right\|_{2,2}=N^{1 / 2}$, and $H_{n}^{2}=N I$. One can easily verify that $\left\|H_{n}\right\|_{1, \infty}=1$ and $\left\|H_{n}\right\|_{1,1}=\|H\|_{\infty, \infty}=N$.

TheOREM 5.4. If $1<p \leq 2 \leq q<\infty$, then the operator $U$ defined by (6) has the following properties:

(i) $\|U\|_{p, q}=1$.

(ii) $U$ is not compact.

(iii) If $p^{\prime} \neq q$ then $U$ is FSS.

(iv) Let $p \leq r \leq q$. Then $U$ factors through $\ell_{r}$ when $p \leq r \leq q^{\prime}$ or $p^{\prime} \leq r \leq q$; otherwise $U \notin \mathcal{J}^{\ell_{r}}$.

(v) In particular, if $p \neq q$ then $U \notin \mathcal{J}^{\ell_{2}}$.

REMARK 5.5. In Section 6 we treat (iii) in the much harder case when $p^{\prime}=q$ and show that in this case $U$ is still FSS.

Proof of Theorem 5.4. Using the Riesz-Thorin interpolation theorem (e.g., [BL76, LT79]) for $H_{n}$ acting as an operator in $L\left(\ell_{1}, \ell_{\infty}\right)$ and as an operator in $L\left(\ell_{2}, \ell_{2}\right)$, and using Remark 5.3, we obtain $\left\|H_{n}\right\|_{r, r^{\prime}} \leq N^{1 / r^{\prime}}$ whenever $1 \leq r \leq 2$. Similarly, interpolating between $\|H\|_{1,1}$ and $\left\|H_{n}\right\|_{2,2}$, and between $\left\|H_{n}\right\|_{2,2}$ and $\|H\|_{\infty, \infty}$, we obtain $\|H\|_{r, r} \leq N^{1 / \min \left\{r, r^{\prime}\right\}}$ whenever $1 \leq r \leq \infty$.

Define $U_{n}^{(r)}=N^{-1 / r^{\prime}} H_{n}$ and $U^{(r)}=\bigoplus_{n=1}^{\infty} U_{n}^{(r)}$; then $\left\|U_{n}^{(r)}\right\|_{r, r^{\prime}} \leq 1$ for every $n$, hence $\left\|U^{(r)}\right\|_{r, r^{\prime}} \leq 1$. Considering $U$ as an operator in $L\left(\ell_{p}, \ell_{q}\right)$, we 
can write

$$
U= \begin{cases}\ell_{p} \stackrel{U^{(p)}}{\longrightarrow} \ell_{q} & \text { when } p^{\prime}=q, \\ \ell_{p} \stackrel{U^{(p)}}{\longrightarrow} \ell_{p^{\prime}} \stackrel{I_{p^{\prime}, q}}{\longrightarrow} \ell_{q} & \text { when } p^{\prime}<q, \\ \ell_{p} \stackrel{I_{p, q^{\prime}}}{\longrightarrow} \ell_{q^{\prime}} \stackrel{U^{\left(q^{\prime}\right)}}{\longrightarrow} \ell_{q} & \text { when } p<q^{\prime} .\end{cases}
$$

It follows immediately that $\|U\|_{p, q} \leq 1$. Since $\mathcal{J}^{\text {FSS }}$ is an ideal, (iii) follows from Proposition 3.3. It also follows from (7) that $U$ factors through $\ell_{r}$ if $p \leq r \leq q^{\prime}$ or $p^{\prime} \leq r \leq q$,

Consider first the case $p^{\prime} \leq q$. Then $U_{n}=N^{-1 / p^{\prime}} H_{n}$. Let $h_{n, i}=H_{n} e_{i}$, the $i$ th column of the $n$th Hadamard matrix. It follows from $H_{n}^{2}=N I$ that $U_{n} h_{n, i}=N^{-1 / p^{\prime}} H_{n}^{2} e_{i}=N^{1 / p} e_{i}$. Thus, $\left\|U_{n} h_{n, i}\right\|_{q}=N^{1 / p}=\left\|h_{n, i}\right\|_{p}$, so that $\left\|U_{n}\right\|_{p, q}=1$. Hence, $U$ is not compact, and $\|U\|_{p, q}=1$ by Remark 2.2.

Next, suppose that $p<r<p^{\prime} \leq q$. We use Corollary 5.2 to show that $U \notin \mathcal{J}^{\ell_{r}}$ in this case. Indeed, assume to the contrary that $U \in \mathcal{J}^{\ell_{r}}$. Then there exists $\widetilde{U}$ such that $\|U-\widetilde{U}\|<1 / 2$ and $\widetilde{U}$ factors through $\ell_{r}$. Let $C$ be the $\ell_{r}$-factorization constant of $\widetilde{U}$. Since $p<\min \left\{r, r^{\prime}\right\}$ one can choose $n$ so that $C<\frac{1}{2} N^{1 / p-1 / \min \left\{r, r^{\prime}\right\}}$, where $N=2^{n}$. Let $\widetilde{U}_{n}$ be the $N \times N$ submatrix of $\widetilde{U}$ corresponding to the $n$th block of $U$, that is, $\widetilde{U}_{n}=Q_{n} \widetilde{U} P_{n}$, where $P_{n}$ (respectively, $Q_{n}$ ) is the canonical projection from $\ell_{p}$ (respectively, $\ell_{q}$ ) onto the span of $e_{N+1}, \ldots, e_{2 N}$. Then the $\ell_{r}$-factorization constant of $\widetilde{U}_{n}$ is at most $C$. It follows from $\left\|U_{n}^{-1} e_{i}\right\|_{p}=\left\|N^{-1 / p} h_{n, i}\right\|_{p}=1$ that

$$
\left\|U_{n}-\widetilde{U}_{n}\right\| \leq\|U-\widetilde{U}\|<1 / 2=\left(2 \max _{1 \leq i \leq N}\left\|U_{n}^{-1} e_{i}\right\|_{p}\right)^{-1}
$$

Let $\delta=\left\|U_{n}^{-1}\right\|_{r^{\prime}, r^{\prime}}$. It follows from $H_{n}^{2}=N I$ and $U_{n}=N^{-1 / p^{\prime}} H_{n}$ that $U_{n}^{-1}=N^{-1 / p} H$, so that

$$
\delta=N^{-1 / p^{\prime}}\left\|H_{n}\right\|_{r^{\prime}, r^{\prime}} \leq N^{-1 / p+1 / \min \left\{r, r^{\prime}\right\}} .
$$

Corollary 5.2 implies that the $\ell_{r}$-factorization constant of $\widetilde{U}_{n}$ is at least $(2 \delta)^{-1} \geq \frac{1}{2} N^{1 / p-1 / \min \left\{r, r^{\prime}\right\}}>C$, which is a contradiction.

The case $p<q^{\prime}$ can be reduced to the previous case by duality. Indeed, it follows from $(7)$ that $U^{*}=I_{q, p^{\prime}} U^{\left(q^{\prime}\right)}: \ell_{q^{\prime}} \rightarrow \ell_{p^{\prime}}$. Consequently, if $p \leq r \leq q^{\prime}$ then $I_{q, p^{\prime}}$ and, therefore, $U^{*}$ factors through $\ell_{r^{\prime}}$. Hence, $U$ factors through $\ell_{r}$. Furthermore, since $H_{n}$ is symmetric for every $n$, it follows that $U_{n}^{*}$ coincides with $U_{n}$ as a matrix and $\left\|U_{n}^{*}\right\|_{q^{\prime}, p^{\prime}}=1$. Applying the previous argument, we observe that $U^{*}$ is non-compact and $\left\|U^{*}\right\|_{q^{\prime}, p^{\prime}}=1$, hence the same is true for $U$. Furthermore, if $q^{\prime}<r<q$, then $U^{*} \notin \mathcal{J}^{\ell_{r^{\prime}}}$ so that $U \notin \mathcal{J}^{\ell_{r}}$.

Finally, (v) follows immediately from (iv).

REMARK 5.6. If $p<r<r^{\prime}<q$ then the operator $\widetilde{U}$ defined as

$$
\ell_{p} \stackrel{I_{p, r}}{\longrightarrow} \ell_{r} \stackrel{U^{(r)}}{\longrightarrow} \ell_{r^{\prime}} \stackrel{I_{r^{\prime}, q}}{\longrightarrow} \ell_{q}
$$


is compact. Indeed, as a matrix

$$
\widetilde{U}_{n}=U_{n}^{(r)}=N^{-1 / r^{\prime}} H_{n}=N^{1 / \min \left\{p^{\prime}, q\right\}-1 / r^{\prime}} U_{n} .
$$

It follows from $\left\|U_{n}\right\|_{p, q}=1$ and $r^{\prime}<\min \left\{p^{\prime}, q\right\}$ that

$$
\left\|\widetilde{U}_{n}\right\|_{p, q}=N^{1 / \min \left\{p^{\prime}, q\right\}-1 / r^{\prime}} \rightarrow 0 \quad \text { as } n \rightarrow 0 .
$$

REMARK 5.7. It follows from Theorem 5.4(iv) that $\mathcal{J}^{\ell_{r}}$ is proper when $\max \left\{p, q^{\prime}\right\}<r<\min \left\{p^{\prime}, q\right\}$. In particular, $\mathcal{J}^{\ell_{2}}$ is proper. It follows from Remark 4.9 and Theorem 5.4(iv) that $\mathcal{J}^{\ell_{2}} \subsetneq \mathcal{J}^{\ell_{r}}$ whenever $p<r<q^{\prime}$ or $p^{\prime}<r<q$. We do not know, however, whether $\mathcal{J}^{\ell_{r}}$ is proper in this case.

Next, we show that if $U^{\prime}$ is another " $U$-like" operator then $U$ and $U^{\prime}$ factor through each other.

Again, we view $\ell_{p}=\left(\bigoplus_{n=1}^{\infty} X_{n}\right)_{p}$ and $\ell_{q}=\left(\bigoplus_{n=1}^{\infty} Y_{n}\right)_{q}$, where $X_{n}=\ell_{p}^{2^{n}}$ and $Y_{n}=\ell_{q}^{2^{n}}$. Denote the basis vectors in $X_{n}$ and $Y_{n}$ by $e_{1}^{(n)}, \ldots, e_{2^{n}}^{(n)}$ and $f_{1}^{(n)}, \ldots, f_{2^{n}}^{(n)}$, respectively. We can view $H_{n}$ and $U_{n}$ as operators from $X_{n}$ to $Y_{n}$.

THEOREM 5.8. Suppose that $\left(n_{i}\right)$ is an increasing sequence, and let $\widetilde{U}=$ $\bigoplus_{i=1}^{\infty} U_{n_{i}}$, viewed as an operator from $\ell_{p}=\left(\bigoplus_{i=1}^{\infty} X_{n_{i}}\right)_{p}$ to $\ell_{q}=\left(\bigoplus_{i=1}^{\infty} Y_{n_{i}}\right)_{q}$. Then $U$ and $\widetilde{U}$ factor through each other.

Proof. Consider the following diagram:

$$
\ell_{p}=\left(\bigoplus_{i=1}^{\infty} X_{n_{i}}\right)_{p} \stackrel{\imath}{\hookrightarrow}\left(\bigoplus_{n=1}^{\infty} X_{n}\right)_{p} \stackrel{U}{\longrightarrow}\left(\bigoplus_{n=1}^{\infty} Y_{n}\right)_{q} \stackrel{R}{\longrightarrow}\left(\bigoplus_{i=1}^{\infty} Y_{n_{i}}\right)_{q}=\ell_{q},
$$

where $\imath$ is the canonical embedding, and $R$ is the canonical projection. We can view $\imath$ and $R$ as operators on $\ell_{p}$ and $\ell_{q}$, respectively. Thus, we get $\widetilde{U}=R U \imath$.

Next, we prove that $U$ factors through $\widetilde{U}$. First, we show that whenever $n<m$ then there exist operators $C: X_{n} \rightarrow X_{m}$ and $D: Y_{m} \rightarrow Y_{n}$ such that $U_{n}=D U_{m} C$ and $\|C\|_{p, p} \leq 1$ and $\|D\|_{q, q} \leq 1$.

First, we consider the case $q \leq p^{\prime}$. Define $C_{n}: X_{n} \rightarrow X_{n+1}$ via $C_{n} e_{i}^{(n)}=$ $e_{i}^{(n+1)}$ for $i=1, \ldots, 2^{n}$. Clearly, $C_{n}$ is an isometry.

Let $Z_{n}$ be the subspace of $Y_{n+1}$ consisting of all the vectors whose first half coordinates are equal to the last half coordinates, respectively, that is, $Z_{n}=\operatorname{span}\left\{f_{i}^{(n+1)}+f_{i+2^{n}}^{(n+1)} \mid i=1, \ldots, 2^{n}\right\}$. Let $P_{n}$ be the "averaging" projection from $Y_{n+1}$ onto $Z_{n}$ given by

$$
P_{n}\left(\sum_{i=1}^{2^{n+1}} \alpha_{i} f_{i}^{(n+1)}\right)=\sum_{i=1}^{2^{n}} \frac{\alpha_{i}+\alpha_{i+2^{n}}}{2}\left(f_{i}^{(n+1)}+f_{i+2^{n}}^{(n+1)}\right) .
$$

Then $\left\|P_{n}\right\|=1$. 
Define $B_{n}: Z_{n} \rightarrow Y_{n}$ via $B_{n}\left(f_{i}^{(n+1)}+f_{i+2^{n}}^{(n+1)}\right)=2^{1 / q} f_{i}^{(n)}$; then $B_{n}$ is an isometry. Hence, $D_{n}=B_{n} P_{n}: Y_{n+1} \rightarrow Y_{n}$ is of norm one.

Fix $1 \leq i \leq 2^{n}$. Since $C_{n} e_{i}^{(n)}=e_{i}^{(n+1)}, H_{n+1} C_{n} e_{i}^{(n)}$ is the $i$ th column of $H_{n+1}$. Since $i \leq 2^{n}$ it follows from the construction of $H_{n}$ 's that the $i$ th column of $H_{n+1}$ is exactly the $i$ th column of $H_{n}$ repeated twice. In particular, $H_{n+1} C_{n} e_{i}^{(n)} \in Z_{n}$ and, therefore, $H_{n+1} C_{n} e_{i}^{(n)}=P_{n} H_{n+1} C_{n} e_{i}^{(n)}$. Finally,

$$
B_{n} P_{n} H_{n+1} C_{n} e_{i}^{(n)}=2^{1 / q}\left(\text { the } i \text { th column of } H_{n}\right)=2^{1 / q} H_{n} e_{i}^{(n)} .
$$

Consequently, we have $D_{n} H_{n+1} C_{n}=2^{1 / q} H_{n}$. It follows from $H_{n}=2^{n / q} U_{n}$ that $D_{n} U_{n+1} C_{n}=U_{n}$. Iterating this $m-n$ times, we get $D U_{m} C=U_{n}$ where $C: X_{n} \rightarrow X_{m}$ is an isometry, and $D: Y_{m} \rightarrow Y_{n}$ is of norm one.

If $q \geq p^{\prime}$, then we consider the adjoint operators. Note that $U_{n}^{*}=U_{n}$ as matrices. Applying the previous argument we find matrices $C$ and $D$ such that $U_{n}^{*}=D U_{m}^{*} C$ with $\|C\|_{q^{\prime}, q^{\prime}} \leq 1$ and $\|D\|_{p^{\prime}, p^{\prime}} \leq 1$. Then $U_{n}=C^{*} U_{m} D^{*}$ is a required factorization in the case $q \geq p^{\prime}$.

It follows that for every $i$ we have

$$
\widetilde{D}_{i} U_{n_{i}} \widetilde{C}_{i}=U_{i}
$$

for some contractions $\widetilde{C}_{i}: X_{i} \rightarrow X_{n_{i}}$ and $\widetilde{D}_{i}: X_{n_{i}} \rightarrow X_{i}$. Let

$$
\begin{aligned}
& \widetilde{C}=\bigoplus_{i=1}^{\infty} \widetilde{C}_{i}:\left(\bigoplus_{i=1}^{\infty} X_{i}\right)_{p} \rightarrow\left(\bigoplus_{i=1}^{\infty} X_{n_{i}}\right)_{p}, \\
& \widetilde{D}=\bigoplus_{i=1}^{\infty} \widetilde{D}_{i}:\left(\bigoplus_{i=1}^{\infty} X_{n_{i}}\right)_{q} \rightarrow\left(\bigoplus_{i=1}^{\infty} X_{i}\right)_{q} .
\end{aligned}
$$

Then $\widetilde{C}: \ell_{p} \rightarrow \ell_{p}$ and $\widetilde{D}: \ell_{q} \rightarrow \ell_{q}$ are bounded, and by (8) we deduce that $\widetilde{D} \widetilde{U} \widetilde{C}=U$.

It follows that any two operators of type $\widetilde{U}$ generated by different sequences factor through each other.

6. The operator $U$ is FSS. Again, let $U$ be the operator defined by (6). Theorem 5.4(iii) states that $U$ is FSS when $p \neq q^{\prime}$. We will show in this section that $U$ is still FSS when $1<p=q^{\prime}$. The argument requires some preparation.

Recall that the $n$th $s$-number of an operator $T \in L(H)$ on a Hilbert space $H$ is defined via $s_{n}(T)=\inf \{\|T-R\| \mid \operatorname{rank} R<n\}$. For $1 \leq r<\infty$, the Schatten norm $\|T\|_{S_{r}}$ of $T$ equals the $\ell_{r}$ norm of the sequence of the $s$-numbers. We say that $T$ belongs to the Schatten class $S_{r}$ if $\|T\|_{S_{r}}<\infty$. We denote by $S_{\infty}$ the set of all compact operators equipped with the operator norm. 
Lemma 6.1. If $T \in L(H)$ is such that $\|T\|_{S_{q}}=1$ and $\inf _{x \in F,\|x\|=1}\|T x\|$ $\geq \varepsilon$ for a subspace $F$ of $H$, then $\operatorname{dim} F \leq \varepsilon^{-q}$.

Proof. Suppose that $\operatorname{dim} F=k$. For every operator $S$ of rank $k-1$ there exists $x \in F$ such that $\|x\|=1$ and $S x=0$. It follows that $\|T-S\| \geq$ $\|T x\| \geq \varepsilon$, so that $s_{1} \geq \cdots \geq s_{k} \geq \varepsilon$. Therefore, $1=\|T\|_{S_{q}}^{q} \geq k \varepsilon^{q}$. Hence $k \leq \varepsilon^{-q}$.

We will also utilize the following result of Maurey [Maur74, Corollary 11, p. 21].

TheOrem 6.2. Let $(\Omega, \mu)$ be a measure space, $Y$ a Banach space, $0<$ $u \leq v<\infty, 1 / u=1 / v+1 / r, T$ a bounded operator from a closed subspace $E$ of $L_{v}(\mu)$ to $Y$, and $C>0$. Then the following are equivalent:

(i) There exists a closed subspace $F$ of $L_{u}(\mu)$ such that $T$ factors as $T=$ $V M_{g}$, where $V: F \rightarrow Y$ with $\|V\| \leq C$, and $M_{g}: L_{v}(\mu) \rightarrow L_{u}(\mu)$ is a multiplication operator defined by $M_{g} f=g f$ for every $f \in L_{v}(\mu)$, with $g \in L_{r}(\mu)$ and $\|g\|_{r} \leq 1$.

(ii) For any $x_{1}, \ldots, x_{n}$ in $E$,

$$
\left(\sum_{i=1}^{n}\left\|T x_{i}\right\|^{u}\right)^{1 / u} \leq C\left[\int\left(\sum_{i=1}^{n}\left|x_{i}\right|^{u}\right)^{v / u} d \mu\right]^{1 / v} .
$$

In what follows, $K_{G}$ will denote the so-called Grothendieck constant, a fundamental constant in Banach space theory (see [DJT95, Tom89, LT77] for details).

Corollary 6.3. Let $(\Omega, \mu)$ be a measure space. Suppose that $q=p^{\prime}$ and $1 / p=1 / 2+1 / r$.

(i) If $T: L_{q}(\mu) \rightarrow \ell_{2}^{k}$ then $T$ can be factored through a multiplication operator from $L_{q}(\mu)$ to $L_{2}(\mu)$, i.e., $T=S M_{g}$, where $S: L_{2}(\mu) \rightarrow \ell_{2}^{k}$ with $\|S\| \leq K_{G}\|T\|$ and $\|g\|_{r}=1$.

(ii) If $T: \ell_{2}^{k} \rightarrow L_{p}(\mu)$ then $T$ can be factored through a multiplication operator from $L_{2}(\mu)$ to $L_{p}(\mu)$, i.e., $T=M_{h} S$, where $S: \ell_{2}^{k} \rightarrow L_{2}(\mu)$ with $\|S\| \leq K_{G}\|T\|$ and $\|h\|_{r} \leq 1$.

Proof. Suppose that $T: L_{q}(\mu) \rightarrow \ell_{2}^{k}$. We verify that condition (ii) of Theorem 6.2 holds for $u=2, v=q=p^{\prime}$, and $r>1$ such that $1 / p=1 / 2+1 / r$ (which is equivalent to $1 / 2=1 / v+1 / r$ ). Let $f_{1}, \ldots, f_{n} \in L_{q}$. Then

$$
\sum_{i=1}^{n}\left\|T f_{i}\right\|^{2}=\sum_{i=1}^{n} \sum_{j=1}^{k}\left|\left(T f_{i}\right)_{j}\right|^{2}=\sum_{j=1}^{k} \sum_{i=1}^{n}\left|\left(T f_{i}\right)_{j}\right|^{2}=\left\|\left(\sum_{i=1}^{n}\left|T f_{i}\right|^{2}\right)^{1 / 2}\right\|_{\ell_{2}}^{2},
$$

where the last expression is the norm of the sequence $\left(\left(\sum_{i=1}^{n}\left|\left(T f_{i}\right)_{j}\right|^{2}\right)^{\frac{1}{2}}\right)_{j=1}^{n}$. It follows from [LT79, Theorem 1.f.14] that 


$$
\begin{aligned}
\left\|\left(\sum_{i=1}^{n}\left|T f_{i}\right|^{2}\right)^{1 / 2}\right\|_{\ell_{2}} & \leq K_{G}\|T\|\left\|\left(\sum_{i=1}^{n}\left|f_{i}\right|^{2}\right)^{1 / 2}\right\|_{L_{q}} \\
& =K_{G}\|T\|\left[\int\left(\sum_{i=1}^{n}\left|f_{i}\right|^{2}\right)^{q / 2} d \mu\right]^{1 / q} .
\end{aligned}
$$

Now (i) follows from Theorem 6.2. To prove (ii), apply (i) to $T^{*}$.

For $N \in \mathbb{N}$ and $1 \leq p \leq \infty$, by $L_{p}^{N}$ we denote the space $L_{p}(\mu)$ where $\mu$ is the uniform probability measure on $\Omega=\{1, \ldots, N\}$. Thus, $L_{p}^{N}=$ $\left(\mathbb{R}^{N},\|\cdot\|_{L_{p}^{N}}\right)$ where, for $\bar{x}=\left(x_{i}\right) \in \mathbb{R}^{N},\|\bar{x}\|_{L_{p}^{N}}=\left(N^{-1} \sum_{i=1}^{N}\left|x_{i}\right|^{p}\right)^{1 / p}$ for $p<\infty$ and $\|\bar{x}\|_{L_{\infty}^{N}}=\max _{1 \leq i \leq N}\left|x_{i}\right|$. Clearly, $\|\cdot\|_{L_{p}^{N}}$ is a scalar multiple of $\|\cdot\|_{\ell_{p}^{N}}$.

The following easy lemma is well-known to specialists. We state it exactly in the form required later and we provide a short proof.

LEMMA 6.4. Consider a product of three operators

$$
S: L_{2}^{N} \stackrel{M_{\psi}}{\longrightarrow} L_{1}^{N} \stackrel{T}{\rightarrow} \ell_{\infty}^{N} \stackrel{D}{\longrightarrow} \ell_{2}^{N}
$$

where $D=\operatorname{diag}\left(d_{j}\right)_{j=1}^{N}$, i.e., the diagonal operator with diagonal $\left(d_{j}\right)$. Then the Hilbert-Schmidt norm of $S$ satisfies $\|S\|_{\mathrm{HS}} \leq\|\psi\|_{L_{2}^{N}}\|T\|\left\|\left(d_{j}\right)\right\|_{\ell_{2}^{N}}$.

Proof. Observe that, in the notation of function spaces on $(\Omega, \mu)$,

$$
S: f \mapsto \psi f \mapsto\left(\left\langle g_{n}, \psi f\right\rangle\right)_{n=1}^{N} \mapsto\left(d_{n}\left\langle g_{n}, \psi f\right\rangle\right)_{n=1}^{N}
$$

for $f \in L_{2}^{N}$ and for some sequence $\left(g_{n}\right)_{n=1}^{N}$ in $L_{\infty}^{N}$, so that $\|T\|=\sup _{n}\left\|g_{n}\right\|_{L_{\infty}^{N}}$. (Here $\langle\cdot, \cdot\rangle$ denotes the inner product with respect to $\mu$.) Let $\left(f_{i}\right)_{i=1}^{N}$ be an orthonormal basis of $L_{2}^{N}$; then

$$
\begin{aligned}
\|S\|_{\mathrm{HS}}^{2} & =\sum_{i=1}^{N}\left\|S f_{i}\right\|_{\ell_{2}^{N}}^{2}=\sum_{i=1}^{N} \sum_{n=1}^{N} d_{n}^{2}\left\langle g_{n}, \psi f_{i}\right\rangle^{2}=\sum_{n=1}^{N} d_{n}^{2} \sum_{i=1}^{N}\left\langle\psi g_{n}, f_{i}\right\rangle^{2} \\
& \left.=\sum_{n=1}^{N} d_{n}^{2}\left\|\psi g_{n}\right\|_{L_{2}^{N}}^{2} \leq\|\psi\|_{L_{2}^{N}}^{2} \sup _{n}\left\|g_{n}\right\|_{L_{\infty}^{N}}^{2}\right)\left\|\left(d_{j}\right)\right\|_{\ell_{2}^{N}}^{2} \cdot \mathbf{~}
\end{aligned}
$$

Theorem 6.5 ([Pis04]). Suppose that $T: L_{p}^{N} \rightarrow \ell_{q}^{N}$ for some $1 \leq p<2$ and $q=p^{\prime}$. Let $E$ be a $k$-dimensional subspace of $L_{p}^{N}$, and $C_{1}, C_{2}$, and $C_{3}$ be positive constants such that

(i) $\|T\|_{L_{2}^{N}, \ell_{2}^{N}} \leq 1$ and $\|T\|_{L_{1}^{N}, \ell_{\infty}^{N}} \leq 1$;

(ii) $E$ is $C_{1}$-isomorphic to $\ell_{2}^{k}$;

(iii) $F=T(E)$ is $C_{2}$-complemented in $\ell_{q}^{N}$;

(iv) $T_{\mid E}$ is invertible and $\left\|\left(T_{\mid E}\right)^{-1}\right\| \leq C_{3}$.

Then $k \leq\left(C_{1}^{3} C_{2} C_{3}^{2} K_{G}^{2}\right)^{q}$. 
Proof. Suppose that $T, E$, and $F$ satisfy the hypotheses for some $C_{1}, C_{2}$, and $C_{3}$. Let $r$ be such that $1 / p=1 / 2+1 / r$. There exists an isomorphism $V: \ell_{2}^{k} \rightarrow E$ such that $\|V\| \leq 1$ and $\left\|V^{-1}\right\| \leq C_{1}$. By Corollary 6.3(ii), $V$ factors through $L_{2}^{N}$. Namely, $V=M_{g} S$ with $S: \ell_{2}^{k} \rightarrow L_{2}^{N}$ such that $\|S\| \leq C_{1} K_{G}$ and $\|g\|_{r} \leq 1$. Let $J: E \rightarrow L_{p}^{N}$ be the canonical inclusion map. We have the diagram

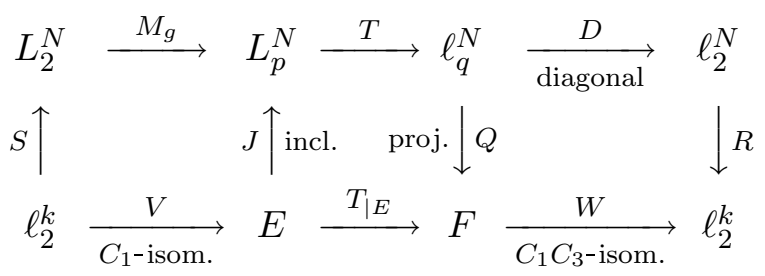

Let $Q$ be a projection from $\ell_{q}^{N}$ onto $F$ with $\|Q\| \leq C_{2}$. It follows from (i) that $\|T\|_{L_{p}^{N}, \ell_{q}^{N}} \leq 1$. Then $F$ is $C_{1} C_{3}$-isomorphic to $\ell_{2}^{k}$. Let $W: F \rightarrow \ell_{2}^{k}$ be an isomorphism such that $\|W\| \leq 1$ and $\left\|W^{-1}\right\| \leq C_{1} C_{3}$. Corollary 6.3(i) implies that $W Q$ factors through $\ell_{2}^{N}$, that is, $W Q=R D$ where $R: \ell_{2}^{N} \rightarrow \ell_{2}^{k}$ with $\|R\| \leq K_{G}\|W Q\| \leq C_{2} K_{G}$, and $D$ is a multiplication (or diagonal) operator $D=\operatorname{diag}\left(d_{j}\right)_{j=1}^{N}$ with $\left\|\left(d_{j}\right)\right\|_{\ell_{r}^{N}} \leq 1$.

We are going to show that $\left\|D T M_{g}\right\|_{S_{q}^{N}} \leq 1$, using the classical complex interpolation argument (see, e.g., [BL76]). For the convenience of the reader not familiar with the subject, we provide the details. Let $Z=\{z \in \mathbb{C} \mid 0 \leq$ $\operatorname{Re} z \leq 1\}$, and define a function $F$ from $Z$ to the unit ball $B\left(L_{2}^{N}, \ell_{2}^{N}\right)$ of $L\left(L_{2}^{N}, \ell_{2}^{N}\right)$ as follows:

$$
F(z)=|D|^{(1-z) r / 2} \operatorname{sign} D T M_{|g|^{(1-z) r / 2} \operatorname{sign} g} .
$$

Here, as usual, $|D|=\operatorname{diag}\left(\left|d_{j}\right|\right)$ and $\operatorname{sign} D=\operatorname{diag}\left(\operatorname{sign} d_{j}\right)$. Observe that $F$ is analytic in the interior of $Z$ as a function from $Z$ to $\mathbb{C}^{N} \times \mathbb{C}^{N}$. Furthermore, $F$ is continuous and bounded on $Z$. A direct calculation shows that if $1 / r=$ $(1-\theta) / 2$ then $F(\theta)=D T M_{g}$.

If $\operatorname{Re} z=1$, it follows from (9) that $F(1+i t)=A_{t} T B_{t}$, where $A_{t}=$ $|D|^{-i t r / 2} \operatorname{sign} D$ and $B_{t}=M_{|g|^{-i t r / 2} \operatorname{sign} g}$. Notice that $A_{t}$ and $B_{t}$ viewed as operators from $\ell_{2}^{N}$ to $\ell_{2}^{N}$ and from $L_{2}^{N}$ to $L_{2}^{N}$ respectively are contractions. It follows that

$$
\|F(z)\|_{L_{2}^{N}, \ell_{2}^{N}} \leq\|T\|_{L_{2}^{N}, \ell_{2}^{N}} \leq 1 \quad \text { whenever } \operatorname{Re} z=1 .
$$

If $\operatorname{Re} z=0$ then we can write

$$
F(i t)=A_{t}|D|^{r / 2} T M_{|g|^{r / 2}} B_{t} .
$$

It can be easily verified that $\left\||g|^{r / 2}\right\|_{L_{2}^{N}} \leq 1$ and $\left\|\left(\left|d_{i}\right|^{r / 2}\right)\right\|_{\ell_{2}^{N}} \leq 1$. Since $\|T\|_{L_{1}^{N}, \ell_{\infty}^{N}} \leq 1$, it follows by Lemma 6.4 that

$$
\|F(z)\|_{\mathrm{HS}} \leq 1 \quad \text { whenever } \operatorname{Re} z=0 .
$$


Put $S_{q}^{N}=S_{q}\left(L_{2}^{N}, \ell_{2}^{N}\right)$. It is known (see, e.g., [GK65, Theorem 13.1]) that the Schatten classes interpolate like $L_{p}$-spaces. Since

$$
\frac{1}{\infty}(1-\theta)+\frac{1}{2} \theta=\frac{1}{2}-\frac{1}{r}=\frac{1}{q}
$$

it follows that $\left(S_{\infty}^{N}, S_{2}^{N}\right)_{\theta}=S_{q}^{N}$.

On the other hand, by definition of a complex interpolation space,

$$
\begin{aligned}
B_{\left(S_{\infty}^{N}, S_{2}^{N}\right)_{\theta}}=\{f(\theta) \mid f: Z & \rightarrow B\left(L_{2}^{N}, \ell_{2}^{N}\right) \text { analytic, } \\
& \left.\left\|f_{\mid\{\operatorname{Re} z=0\}}\right\|_{S_{2}} \leq 1 \text { and }\left\|f_{\mid\{\operatorname{Re} z=1\}}\right\|_{S_{\infty}} \leq 1\right\} .
\end{aligned}
$$

Since $\|\cdot\|_{S_{2}}=\|\cdot\|_{\mathrm{HS}}$ and $\|\cdot\|_{S_{\infty}}=\|\cdot\|_{L_{2}^{N}, \ell_{2}^{N}}$, it follows from (10) and (11) that $D T M_{g}=F(\theta) \in B_{\left(S_{\infty}^{N}, S_{2}^{N}\right)_{\theta}}$ and, thus, $\left\|D T M_{g}\right\|_{S_{q}^{N}} \leq 1$. It follows that

$$
\|W T V\|_{S_{q}}=\left\|R D T M_{g} S\right\|_{S_{q}} \leq\|R\|\left\|D T M_{g}\right\|_{S_{q}}\|S\| \leq C_{1} C_{2} K_{G}^{2} .
$$

Note that $\left\|(W T V)^{-1}\right\| \leq C_{1}^{2} C_{3}^{2}$. It follows from Lemma 6.1 that

$$
k \leq\left(\frac{1}{C_{1}^{2} C_{3}^{2}} \frac{1}{C_{1} C_{2} K_{G}^{2}}\right)^{-q}=\left(C_{1}^{3} C_{2} C_{3}^{2} K_{G}^{2}\right)^{q} .
$$

This concludes the proof.

We also need the following lemma, which generalizes Lemma 3.4. Assume that $X$ is a Banach space with an FDD $\left(X_{n}\right)_{n=1}^{\infty}$ (see [LT77] for the definition of FDD). Let $P_{n}$ be the canonical projection from $X$ onto $X_{n}$, and assume that $X$ satisfies the following condition, which means that $X$ is far from a $c_{0}$-sum of the $X_{n}$ 's:

$$
\begin{aligned}
& \text { for any } \delta>0 \text { there is a } k=k(\delta) \text { in } \mathbb{N} \text { so that whenever } x \in S_{X}, \text { then } \\
& \operatorname{card}\left\{n \in \mathbb{N} \mid\left\|P_{n} x\right\| \geq \delta\right\}<k \text {. }
\end{aligned}
$$

Suppose that for every $n \in \mathbb{N}$ we are given a seminorm $q_{n}$ on $X_{n}$ such that $q_{n}(x) \leq\|x\|$, where $q_{n}(x)$ stands for $q_{n}\left(P_{n} x\right)$ whenever $x \in X$.

Lemma 6.6. Suppose that $X,\left(X_{n}\right)$, and $\left(q_{n}\right)$ are as in the preceding paragraph and $0<r \leq 1$. Then there exists $\varepsilon>0$ such that for every $l \in \mathbb{N}$ there exists $L \in \mathbb{N}$ such that for every $L$-dimensional subspace $G$ of $X$ such that $\max _{n \in \mathbb{N}} q_{n}(x) \geq r\|x\|$ for all $x \in G$ there exists an l-dimensional subspace $F \subseteq G$ and an index $n_{0}$ such that $q_{n_{0}}(x) \geq \varepsilon\|x\|$ for all $x \in F$.

To prove Lemma 6.6 we need the following stabilization result (see, e.g., [MS86, p. 6]).

Theorem 6.7. For every $n \in \mathbb{N}, \varepsilon>0$ and $c>0$ there is an $N=$ $N(n, \varepsilon, c) \in \mathbb{N}$ so that for any $N$-dimensional space $E$, and any Lipschitz map $f: S_{E} \rightarrow \mathbb{R}$ whose Lipschitz constant does not exceed $c$, there is an $n$ dimensional subspace $F$ of $E$ so that

$$
\max \left\{f(x): x \in S_{F}\right\}-\min \left\{f(x): x \in S_{F}\right\} \leq \varepsilon .
$$


Proof of Lemma 6.6. Let $k(\cdot)$ be the function defined in (12). Put

$$
m=k\left(r^{2} / 4\right), \quad \delta=r / 4 m, \quad s=k(\delta) .
$$

It suffices to show that for $l^{\prime} \in \mathbb{N}$ there exists $L$ so that, if $G$ is a subspace of $X$ of dimension $L$ and $\max _{n \in \mathbb{N}} q_{n}(x) \geq r\|x\|$ for all $x \in G$, then $G$ has an $l^{\prime}$-dimensional subspace $F^{\prime}$ and a set $I \subset \mathbb{N}$ with card $I=s$ such that $\max _{n \in I} q_{n}(x) \geq \delta\|x\|$ for all $x \in F^{\prime}$.

Indeed, once we prove this formally weaker claim, we can take a number $l^{\prime}$ large enough so that Theorem 6.7 can be applied $s$ times to deduce that $F^{\prime}$ has an $l$-dimensional subspace $F$ which has the property that, for all $n \in I$,

$$
\max _{x \in S_{F}} q_{n}(x)-\min _{x \in S_{F}} q_{n}(x) \leq \delta / 2 .
$$

Now pick any $y \in S_{F}$; then $q_{n_{0}}(y)=\max _{n \in I} q_{n}(y) \geq \delta$ for some $n_{0} \in I$. Then for every $x \in S_{F}$ we have

$$
q_{n_{0}}(x) \geq \min _{z \in S_{F}} q_{n_{0}}(z) \geq \max _{z \in S_{F}} q_{n_{0}}(z)-\delta / 2 \geq q_{n_{0}}(y)-\delta / 2 \geq \delta / 2,
$$

so that the statement of our lemma is satisfied for $\varepsilon=\delta / 2$.

Let $l^{\prime} \in \mathbb{N}$ and define numbers $L_{0}, L_{1}, \ldots, L_{m}$ as follows. Put $L_{0}=l^{\prime}$, and, assuming that $L_{0}, L_{1}, \ldots, L_{n}, n<m$, have already been defined, use Theorem 6.7 to choose $L_{n+1}$ large enough so that for every $L_{n+1}$-dimensional subspace $G$ of $X$ and every Lipschitz-1 map $f: S_{G} \rightarrow \mathbb{R}$ there is an $L_{n^{-}}$ dimensional subspace $G^{\prime} \subseteq G$ such that

$$
\max _{x \in G^{\prime}} f(x)-\min _{x \in G^{\prime}} f(x) \leq \delta .
$$

Let $L=L_{m}$. Assume that our claim is false. This would mean that there exists a subspace $G$ of $X$ with $\operatorname{dim} G=L$ such that

(14) for each $I \subset \mathbb{N}$ of card $I=s$ and each subspace $F^{\prime} \subseteq G$ of $\operatorname{dim} F^{\prime}=l^{\prime}$ there exists $x \in S_{F^{\prime}}$ such that $\max _{n \in I} q_{n}(x) \leq \delta$.

Choose an arbitrary vector $x_{1} \in S_{G}$ and a subset $I_{1} \subset \mathbb{N}$ with card $I_{1}=s$ so that $\min _{n \in I_{1}} q_{n}\left(x_{1}\right) \geq \max _{n \in \mathbb{N} \backslash I_{1}} q_{n}\left(x_{1}\right)$. It follows from (13) that there exists an index $n_{1}$ such that $q_{n_{1}}\left(x_{1}\right) \geq r$; we can assume that $n_{1} \in I_{1}$. On the other hand, the definition of $s$ implies that $q_{n}\left(x_{1}\right) \leq \delta$ whenever $n \notin I_{1}$. It follows from the definition of $L_{m}$ that there exists a subspace $G_{m-1}$ of $G$ of dimension $L_{m-1}$ so that

$$
\max _{x \in S_{G_{m-1}}} \max _{n \in I_{1}} q_{n}(x) \leq \min _{x \in S_{G_{m-1}}} \max _{n \in I_{1}} q_{n}(x)+\delta \leq 2 \delta,
$$

where the last inequality follows from (14).

Next, pick an $x_{2} \in S_{G_{m-1}}$ and $I_{2} \subset \mathbb{N} \backslash I_{1}$ so that card $I_{2}=s$ and $\min _{n \in I_{2}} q_{n}\left(x_{2}\right) \geq \max _{n \notin I_{1} \cup I_{2}} q_{n}\left(x_{2}\right)$. Again, it follows from (13) that there 
exists an index $n_{2}$ such that $q_{n_{2}}\left(x_{2}\right) \geq r$; we can assume that $n_{2} \in I_{1} \cup I_{2}$. By (15), $q_{n}\left(x_{2}\right) \leq 2 \delta<r$ for each $n \in I_{1}$, so that $n_{2} \in I_{2}$. Again, $q_{n}\left(x_{2}\right) \leq \delta$ whenever $n \notin I_{1} \cup I_{2}$. We can choose a subspace $G_{m-2}$ of $G_{m-1}$ of dimension $L_{m-2}$ so that

$$
\max _{x \in S_{G_{m-2}}} \max _{n \in I_{2}} q_{n}(x) \leq 2 \delta .
$$

Proceeding this way, we obtain a sequence of vectors $x_{1}, \ldots, x_{m}$ and disjoint sets $I_{1}, \ldots, I_{m}$ of cardinality $s$, and indices $n_{1}, \ldots, n_{m}$, such that for each $i=1, \ldots, m$ we have $n_{i} \in I_{i}$ and $q_{n_{i}}\left(x_{i}\right) \geq r$. Also,

$$
q_{n}\left(x_{i}\right) \leq \begin{cases}2 \delta & \text { if } n \in I_{1} \cup \cdots \cup I_{i-1}, \\ \delta & \text { if } n \notin I_{1} \cup \cdots \cup I_{i},\end{cases}
$$

hence $q_{n}\left(x_{i}\right) \leq 2 \delta$ whenever $n \notin I_{i}$. If $n \in I_{i}$ then $q_{n}\left(x_{i}\right) \leq\left\|x_{i}\right\|=1$.

Put $x=\sum_{i=1}^{m} x_{i}$; then for every $n \in \mathbb{N}$ we have $q_{n}(x) \leq 1+m \cdot 2 \delta \leq 2$. On the other hand,

$$
r \leq q_{n_{i}}\left(x_{i}\right) \leq q_{n_{i}}(x)+q_{n_{i}}\left(x-x_{i}\right) \leq q_{n_{i}}(x)+2 m \delta,
$$

so that $q_{n_{i}}(x) \geq r-2 m \delta=r / 2$ for each $i=1, \ldots, m$. It follows from the definition of $m$ that there can be at most $m-1$ indices $n$ such that $q_{n}(x) \geq r^{2}\|x\| / 4$, hence $r^{2}\|x\| / 4>r / 2$. It follows that $\|x\|>2 / r$, so that $q_{n}(x) \leq 2<r\|x\|$ for every $n \in \mathbb{N}$, which is a contradiction.

Now we are ready to prove that $U$ is FSS.

THEOREM 6.8. The operator $U$ constructed in (6) is FSS for all $1<p \leq$ $2 \leq q<\infty$, unless $p=q=2$.

Proof. In view of Theorem 5.4(iii) we may assume that $q=p^{\prime}$. Recall that $U=\bigoplus_{n=1}^{\infty} U_{n}$ is composed of blocks $U_{n}: X_{n} \rightarrow Y_{n}$, where $X_{n}=\ell_{p}^{2^{n}}$ and $Y_{n}=\ell_{q}^{2^{n}}$. For each $n$, let $P_{n}: \ell_{p} \rightarrow X_{n}$ be the canonical projection. For $x \in \ell_{p}$ put $q_{n}(x)=\left\|U_{n} P_{n} x\right\|$. By Theorem 5.4(i) we have $q_{n}(x) \leq\|x\|$.

Assume that $U$ is not FSS. Then there exists a constant $C$ such that there are subspaces $G$ of $\ell_{p}$ of arbitrarily large dimension such that the restriction of $U$ to $G$ is a $C$-isomorphism. Let $x \in S_{G}$, and write $x=\sum_{n=1}^{\infty} x_{n}$ where $x_{n} \in X_{n}$; then $\|U x\| \geq 1 / C$. On the other hand,

$$
\|U x\|^{q}=\sum_{n=1}^{\infty}\left\|U_{n} x_{n}\right\|^{q} \leq \max _{n \in \mathbb{N}}\left\|U_{n} x_{n}\right\|^{q-p} \sum_{n=1}^{\infty}\left\|U_{n} x_{n}\right\|^{p} \leq \max _{n \in \mathbb{N}} q_{n}(x)^{q-p} .
$$

Hence, $\max _{n \in \mathbb{N}} q_{n}(x) \geq C^{q /(p-q)}$.

It follows from Lemma 6.6 that there exists $\varepsilon>0$ such that for every $k$ and for every $G \subseteq \ell_{p}$ of sufficiently large dimension there exists a subspace $F$ of $G$ and an index $n$ such that $\operatorname{dim} F=k$ and $q_{n}(x) \geq \varepsilon$ for all $x \in S_{F}$. This implies that the restriction of $U_{n} P_{n}$ to $F$ is a $1 / \varepsilon$-isomorphism. Put $E=P_{n}(F)$; then $E$ is a $k$-dimensional subspace of $X_{n}$, and $U_{n}$ is a $1 / \varepsilon$ - 
isomorphism on $E$. In view of Theorem 4.10 we may assume that $E$ is 2isomorphic to $\ell_{2}^{k}$ and $U_{n}(E)$ is $K$-complemented in $\ell_{q}^{2^{n}}$.

Let $V_{n}$ be the canonical isometry between $L_{p}^{N}$ and $X_{n}=\ell_{p}^{N}$, where $N=2^{n}$. It follows that $\left\|V_{n} x\right\|_{\ell_{r}^{N}}=N^{1 / r-1 / p}\|x\|_{L_{r}^{N}}$ for every $x \in L_{p}^{N}$ and every $r \in[p, q]$. It follows from the definition of $U_{n}$ and Remark 5.3 that

$$
\begin{aligned}
& \left\|U_{n} V_{n}\right\|_{L_{2}^{N}, \ell_{2}^{N}}=N^{1 / 2-1 / p}\left\|U_{n}\right\|_{\ell_{2}^{N}, \ell_{2}^{N}}=N^{1 / 2-1 / p-1 / q}\left\|H_{n}\right\|_{\ell_{2}^{N}, \ell_{2}^{N}}=1, \\
& \left\|U_{n} V_{n}\right\|_{L_{1}^{N}, \ell_{\infty}^{N}}=N^{1-1 / p}\left\|U_{n}\right\|_{\ell_{1}^{N}, \ell_{\infty}^{N}}=N^{1-1 / p-1 / q}\left\|H_{n}\right\|_{\ell_{1}^{N}, \ell_{\infty}^{N}}=1 .
\end{aligned}
$$

Now applying Theorem 6.5 to $U_{n} V_{n}$ and $V_{n}^{-1}(E)$ we obtain a contradiction with the fact that $k=\operatorname{dim} E$ was chosen arbitrarily.

REMARK 6.9. If $p=q=2$ then $U$ is an isometry, hence not FSS. Consider the case when $p=1$ and $q=\infty$. The preceding proof does not work, since now we cannot use Theorem 4.10. Actually, $U$ is not FSS in this case. Indeed, we now have $U_{n}=H_{n}$. It is easy to see that among the columns of $H_{n}$ one finds all the Rademacher vectors (of length $N=2^{n}$ ). Since the span of these vectors in $\ell_{\infty}^{N}$ is isometrically isomorphic to $\ell_{1}^{n}$, it follows that the restriction of $H_{n}$ to the appropriate subspace of $\ell_{1}^{N}$ preserves a copy of $\ell_{1}^{n}$.

Question. Are there any other closed ideals in $L\left(\ell_{p}, \ell_{q}\right)$ ? In view of the diagram at the beginning of our paper this question can be subdivided into the following subquestions:

(i) Is $\mathcal{J}^{I_{p, q}}$ equal to $\mathcal{J}^{\text {FSS }} \cap \mathcal{J}^{\ell_{2}}$ ? If not, is $\mathcal{J}^{\text {FSS }} \cap \mathcal{J}^{\ell_{2}}$ an immediate successor of $\mathcal{J}^{I_{p, q}}$ ?

(ii) Is $\mathcal{J}^{\text {FSS }}$ an immediate successor of $\mathcal{J}^{\text {FSS }} \cap \mathcal{J}^{\ell_{2}}$ ? More generally, are there any immediate successors of $\mathcal{J}^{\mathrm{FSS}} \cap \mathcal{J}^{\ell_{2}}$ other than $\mathcal{J}^{\ell_{2}}$ ?

(iii) Is $\mathcal{J}^{\text {FSS }} \vee \mathcal{J}^{\ell_{2}}$ an immediate successor of $\mathcal{J}^{\ell_{2}}$ ?

(iv) Is $\mathcal{J}^{\mathrm{FSS}} \vee \mathcal{J}^{\ell_{2}}$ equal to $L\left(\ell_{p}, \ell_{q}\right)$ ?

Question. Suppose again that $U$ is the operator defined in (6). Since $U$ is FSS, we have $\mathcal{J}^{U} \subseteq \mathcal{J}^{\text {FSS }}$. Does $\mathcal{J}^{U}$ equal $\mathcal{J}^{\text {FSS}}$ ?

\section{References}

[BL76] J. Bergh and J. Löfström, Interpolation Spaces. An Introduction, Grundlehren Math. Wiss. 223, Springer, Berlin, 1976.

[Calk41] J. W. Calkin, Two-sided ideals and congruences in the ring of bounded operators in Hilbert space, Ann. of Math. (2) 42 (1941), 839-873.

[CPY74] S. R. Caradus, W. E. Pfaffenberger, and B. Yood, Calkin Algebras and Algebras of Operators on Banach Spaces, Lecture Notes in Pure and Appl. Math. 9, Dekker, New York, 1974.

[Daws06] M. Daws, Closed ideals in the Banach algebra of operators on classical nonseparable spaces, Math. Proc. Cambridge Philos. Soc. 140 (2006), 317-332.

[DJT95] J. Diestel, H. Jarchow, and A. Tonge, Absolutely Summing Operators, Cambridge Stud. Adv. Math. 43, Cambridge Univ. Press, Cambridge, 1995. 
[FT79] T. Figiel and N. Tomczak-Jaegermann, Projections onto Hilbertian subspaces of Banach spaces, Israel J. Math. 33 (1979), 155-171.

[GK65] I. C. Gohberg [I. Ts. Gokhberg] and M. G. Kreı̆n, Introduction to the Theory of Linear Non-Selfadjoint Operators in Hilbert Space, Nauka, Moscow, 1965 (in Russian); English transl.: Amer. Math. Soc., Providence, RI, 1969.

[GMF60] I. C. Gohberg [I. Ts. Gokhberg], A. S. Markus, and I. A. Fel'dman, Normally solvable operators and ideals associated with them, Bul. Akad. Stiince RSS Moldoven. 1960, no. 10 (76), 51-70 (in Russian); English transl.: Amer. Math. Soc. Transl. 61 (1967), 63-84.

[Gram67] B. Gramsch, Eine Idealstruktur Banachscher Operatoralgebren, J. Reine Angew. Math. 225 (1967), 97-115.

[LLR04] N. J. Laustsen, R. J. Loy, and C. J. Read, The lattice of closed ideals in the Banach algebra of operators on certain Banach spaces, J. Funct. Anal. 214 (2004), 106-131.

[LSZ06] N. J. Laustsen, Th. Schlumprecht, and A. Zsák, The lattice of closed ideals in the Banach algebra of operators on a certain dual Banach space, J. Operator Theory 56 (2006), 391-402.

[LT77] J. Lindenstrauss and L. Tzafriri, Classical Banach Spaces. I, Springer, Berlin, 1977.

[LT79] -, 一, Classical Banach Spaces. II, Springer, Berlin, 1979.

[Luft68] E. Luft, The two-sided closed ideals of the algebra of bounded linear operators of a Hilbert space, Czechoslovak Math. J. 18 (1968), 595-605.

[Masc94] V. Mascioni, A restriction-extension property for operators on Banach spaces, Rocky Mountain J. Math. 24 (1994), 1497-1507.

[Maur74] B. Maurey, Théorèmes de factorisation pour les opérateurs linéaires à valeurs dans les espaces $L^{p}$, Astérisque 11 (1974).

[Milm70] V. D. Milman, Operators of class $C_{0}$ and $C_{0}^{*}$, Teor. Funktsiǔ Funktsional. Anal. i Prilozhen. 10 (1970), 15-26 (in Russian).

[MS86] V. D. Milman and G. Schechtman, Asymptotic Theory of Finite-Dimensional Normed Spaces, Lecture Notes in Math. 1200, Springer, Berlin, 1986.

[Piet78] A. Pietsch, Operator Ideals, Math. Monogr. 16, Deutscher Verlag Wiss., Berlin, 1978.

[Pis04] G. Pisier, private communications.

[Tom89] N. Tomczak-Jaegermann, Banach-Mazur Distances and Finite-Dimensional Operator Ideals, Pitman Monogr. Surveys Pure Appl. Math. 38, Longman, Harlow, 1989.

Department of Mathematics

University of North Texas

Denton, TX 76203-1430, U.S.A.

E-mail: bunyamin@unt.edu
Department of Mathematics Texas A\&M University College Station, TX 77843-3368, U.S.A. E-mail: schlump@math.tamu.edu

Department of Mathematical and Statistical Sciences

University of Alberta

Edmonton, AB, T6G 2G1, Canada

E-mail: nicole@ellpspace.math.ualberta.ca vtroitsky@math.ualberta.ca 